\title{
The allometric quarter-power scaling model and its applicability to Grand fir and Eucalyptus trees
}

\author{
Hannah Capes · Robert J. Maillardet · Thomas G. Baker · \\ Christopher J. Weston · Don McGuire · Ian C. Dumbrell · \\ Andrew P. Robinson
}

Received: date / Accepted: date

\begin{abstract}
The use of the allometric model $y=\beta x^{\alpha}$ to describe the relative growth of morphological traits of trees is a source of contention in ecology. This is particularly so in a specific form, the West, Brown and Enquist model, which predicts values of $\alpha$ that are multiples of 1/4 for various allometric relationships - the quarter-power scaling law. We use statistical techniques to test the appropriateness of the quarterpower scaling allometric model in a number of different relative growth relationships of trees. Two separate 6 datasets are used, one of repeated measures of Abies grandis (Grand fir) trees, another of independent measures of Eucalyptus trees. Nonlinear mixed-effects modelling is used to fit allometric models to the datasets. Generalized additive models, equivalence testing and traditional significance testing are used to assess the adequacy of the allometric models fitted and the values of the estimated exponents relative to those predicted by the WBE model. In only one of the five models fitted was there empirical evidence for the WBE-predicted quarter-power exponent. However the adequacy of the allometric models was generally supported, though a need for further analysis over a larger range of tree ages/sizes is indicated.
\end{abstract}

Keywords Allometric scaling · quarter-power scaling law · nonlinear mixed-effects models · equivalence testing

Hannah Capes (Email: hannah.capes@sweeneyresearch.com.au), Sweeney Research Melbourne, VIC, Australia 3205; Robert J. Maillardet (Email: rjmail@unimelb.edu.au), The University pf Melbourne (UOM), Parkvillle, VIC, Australia 3052 ; Thomas G. Baker, (Email: tgbaker@unimelb.edu.au), UOM, Richmand, VIC, Australia 3121; Christopher J. Westorł(Email: weston@unimelb.edu.au), UOM, Parkvillle, VIC, Australia 3052; Don McGuire Email: domcguire54@gmail.com), Not affiliated; Ian C. Dumbrell (Email: ian.dumbrell@fpc.wa.gov.au), Forest Products Commission, Kensinfter, WA, Australia 6151; Andrew P. Robinson (Email: apro@unimelb.edu.au), UOM, Parkvillle, VIC, Australia 3052. 
The allometric quarter-power scaling model and its applicability to Grand fir and Eucalyptus trees

Authors de-identified for refereeing

Received: date / Accepted: date

Institutions de-identified for refereeing 
Abstract The use of the allometric model $y=\beta x^{\alpha}$ to describe the relative growth of morphological trait$\mathrm{s}$ of trees is a source of contention in ecology. This is particularly so in a specific form, the West, Brown 3 and Enquist model, which predicts values of $\alpha$ that are multiples of 1/4 for various allometric relationships - the quarter-power scaling law. We use statistical techniques to test the appropriateness of the quarterpower scaling allometric model in a number of different relative growth relationships of trees. Two separate 6 datasets are used, one of repeated measures of Abies grandis (Grand fir) trees, another of independent measures of Eucalyptus trees. Nonlinear mixed-effects modelling is used to fit allometric models to the datasets. Generalized additive models, equivalence testing and traditional significance testing are used to assess the 9 adequacy of the allometric models fitted and the values of the estimated exponents relative to those predicted by the WBE model. In only one of the five models fitted was there empirical evidence for the WBE-predicted quarter-power exponent. However the adequacy of the allometric models was generally supported, though a need for further analysis over a larger range of tree ages/sizes is indicated.

Keywords Allometric scaling · quarter-power scaling law $\cdot$ nonlinear mixed-effects models $\cdot$ equivalence testing 


\section{Introduction: Allometry and the quarter-power scaling law}

The size of an organism and the relative sizes of its morphological components are defining characteristics of the organism in terms of its structure and function. It is often of interest to study such defining characteristics, for instance the relationship between tree mass (a common substitute for 'size') and height, or between tree mass and stem diameter. A commonly applied means of doing so is provided by the allometric equation, introduced by Huxley (1932),

$$
y=\beta x^{\alpha} .
$$

This equation is widely used in biology and ecology to describe allometric relationships. Appeal to the geometric, biological and physiological consequences and constraints of organism magnitude has allowed the allometric exponent $\alpha$ to be explicitly specified for certain relationships. For instance, consideration of gravity led to the specific allometries for the load-bearing limbs of animals of

$$
\begin{gathered}
\text { length } \propto \operatorname{mass}^{1 / 4} \text { and } \\
\text { diameter } \propto \text { mass }^{3 / 8}, \text { leading to } \\
\text { length } \propto \text { diameter }^{2 / 3}
\end{gathered}
$$

under the principle of elastic similarity (Strauss, 1993). These same three relationships are also believed to hold for the weight-bearing limbs of trees as well as animals (Enquist, 2002).

In 2000, West, Brown and Enquist proposed a model through which they sought to provide a unifying theoretical basis for such relationships in trees (vascular plants) (West et al., 2000, Enquist, 2002). The theory models a tree as a hierarchical vascular network which branches through a number of levels to supply the whole tree with water and nutrients, from bole to petioles. Basic principles of hydrodynamics and biomechanics are assumed to apply, including volume filling, size invariance, uniform biomechanical constraints, energy minimisation, and fractal self-similarity and area preservation through branching levels.

This model (referred to as the WBE model hereafter) predicts that many characteristics of trees $Y$ will follow the allometric model $Y=\beta M^{\alpha}$, where $M$ is the dry mass of the tree, and $\alpha$ is a multiple of $1 / 4$, hence commonly referred to as 'quarter-power scaling' relationships (Enquist, 2002).

The coefficient $\beta$ is permitted to vary in accordance with the tree's internal or external circumstances, such as species and age or resource supply and climate respectively (Huxley, 1932; Enquist, 2002). Some specific scaling exponents predicted by the WBE model are summarized in Table 1.

[Table 1 about here.]

There have been quite significant challenges to the assumptions of the WBE model, most based on empirical evidence of tree architecture that contradict the model simplifications, including appearance of leaves at multiple branching levels; lack of invariance for total petiole/leaf size; tapering of resource supply channels within, not just between, model branches, and lack of morphological equivalence of all branch links (Nygren and Pallardy, 2008). The WBE authors have acknowledged the existence of departures from the assumptions 
of their 'zeroth-order model', seeing in them opportunities for more detailed models (West et al., 1999, Enquist 2002). The presence of stem and branch heartwood that plays no part in conducting water has also not been considered by proponents of allometric scaling of trees with WBE-predicted exponents (Mäkela and Valentine, 2006).

There are three situations to which the allometric model and thus the WBE model can be applied (Strauss. 1993):

1. Static allometry — to describe variation among individuals at the same age.

2. Ontogenetic allometry - to describe variation in the individual over time (think of longitudinal time series).

3. Evolutionary allometry - to describe variation among individuals at a mixture of stages of life (think of cross-sectional time series).

In this paper we are concerned with testing the accuracy of the specific exponents predicted by the WBE model, and the ability of the allometric model itself to adequately model tree growth, with respect to static and evolutionary allometry.

Previous work has tackled the same problem using different approaches. Coomes and Allen (2009) used traditional significance testing and standardized major axis regression upon 45 tree species from a Costa Rican forest that had been used by Enquist et al. (1999) to test whether tree stem diameter growth scales with stem diameter to the one-third power, following WBE. Coomes and Allen (2009) concluded that the evidence failed to support WBE, although see Stark et al. (2011), and followed up with a more extensive study that also failed to support WBE (Coomes et al. 2011). Rüger and Condit(2012) applied similar tests to the same model using another dataset and drew similar conclusions, particularly that "the prediction of metabolic ecology for the scaling of tree growth with size is only valid when competition for light is unimportant." .

Here, we test a wider range of allometric relationships between organs, we test more than one kind of allometry, and we apply equivalence testing, which arguably provides a more logical assessment of the model's veracity. The balance of the paper is laid out as follows. We next introduce the two datasets that we use to assess WBE model. We then describe the modelling procedure, including how we test for adequacy of the model implied by WBE and how we apply the equivalence tests to the parameter estimates. We provide results and then a discussion. Supplementary information and detailed supporting analyses are available from the JABES website (the 'AS Supplement'), including access to the datasets that we used.

\section{The data}

We used two datasets to critique the WBE model. The first, the Abies dataset, provided height and stem diameter at $1.37 \mathrm{~m}$ (called dbh in the USA) measurements of trees in varied environmental conditions over time (Stage, 1963). The model fitted to this dataset was used to examine static allometry. The second, the Eucal dataset, provided measurements of such variables as height, stem diameter at $1.30 \mathrm{~m}$ (called dbh 
in Australia), mass, and leaf area, for 492 individual trees. The models fitted to this dataset were used to examine evolutionary allometry for a number of different relationships.

\subsection{The Abies dataset}

The Abies dataset is a set of measurements of height and diameter made on 66 Grand fir trees growing in various locations and habitats over many decades (Stage, 1963). The trees were purposively sampled for quality and lack of suppression from nine national forests in the US: Kaniksu, Coeur d'Alene, St. Joe, Clearwater, Nez Perce, Clark Fork, Umatilla, Wallowa and Payette. Sampling was undertaken by felling the trees, then splitting them in two along the bole and counting growth rings to establish decadal measures of height and dbhub (Diameter at breast height, here $1.37 \mathrm{~m}$, under bark). The trees grew in five different habitats, as characterised by the 'climax tree species' (the most shade-tolerant species that could grow on a particular tree's site) and the 'dominant understory plant'. The species involved were recorded in abbreviated form:

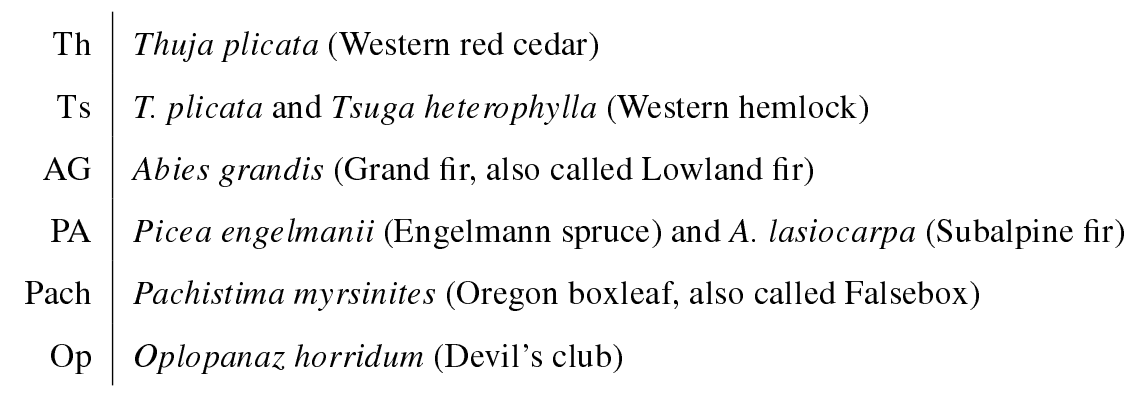

The five habitats (climax tree/dominant understory plant combinations) were ‘Ts/Pach', ‘Ts/Op', 'Th/Pach', 'AG/Pach', 'PA/Pach'. The Abies dataset includes measurements on the following variables: Forest (Forest and Forest.ID), Habitat Type (HabType and Hab.ID), Diameter at $1.37 \mathrm{~m}$ (Dbhub), Height (Height), Age (Age) and decade of measurement increasing from 0 (representing 1963) (Decade).

\subsection{The Eucal dataset}

This dataset comprises single measures made on 492 planted trees (300 Eucalyptus globulus, Tasmanian Blue Gum; 192 E. grandis, Flooded Gum), growing in 19 different sites over southern Australia. Within each site, trees were grown in a number of stands; within each stand were a number of plots, reflecting a range of treatments, including irrigation and fertiliser application (the data arose from a designed experiment that assessed the effect of treatments upon differential organ growth). Here we use only the untreated trees. In addition, for each site (and hence approximately for each tree) we have meteorological measurements including annual rainfall and monthly average minimum and maximum daily air temperatures. The trees were all harvested and measured at specific ages but not necessarily at the same time.

This data set provided a wide range of physiological measurements for these trees (the AS Supplement has details of site locations, data sources, and the full list of variables measured). In the context of testing the 
form and predictions of the WBE model, measurements were available for stem diameter over bark at $1.3 \mathrm{~m}$ (dob1.3), total tree height $(h t)$, and total tree mass $(m)$. All mass measurements were aboveground biomass, defined as the oven-dry $\left(\right.$ c. $\left.70-80^{\circ} \mathrm{C}\right)$ mass of organic matter above the soil including stem, branches, bark and foliage. Among these three dimensions, the WBE model proposed the following relationships (taken from Table 1):

- $M \propto$ Tree diameter $8 / 3$

- Tree height $\propto M^{1 / 4}$.

- Tree height $\propto$ Branch diameter ${ }^{2 / 3}$;

The available data provided a large sample for us to use nonlinear regression to model four relationships. The proportionality of each of these relationships can be examined visually in Figure 1, where the predictors are plotted against their WBE-transformed responses with a loess fitted smooth. The loess fitted smooth has been estimated using least squares methods with a smoothing parameter of 0.1 for each relationship. The transformed relationships seem plausible.

We wished to see whether the best model that we could fit (in terms of both adequacy of regression assumptions and satisfactory prediction performance) would confirm or refute the proposals of the WBE model in terms of the suitability of the allometric model and of its predicted exponents.

[Fig. 1 about here.]

Because each tree from the Eucal dataset contributed a single observation, the observations and hence the residuals were treated as having no time-related correlation at the tree level. However, there may have been similarities between trees grown in common sites, which suggested the use of mixed-effects modelling with site as the grouping factor.

Several transformations were made to the variables involved. The age of each tree in years was known from planting records, but we used age $1=0.1 \times$ age to enable an interpretation in terms of change in dimension by decade instead of year. The variable stocking was provided in units of trees/hectare. A rearrangement that enabled discussion of the variable in terms more immediately relevant to the individual trees and sites was stocking $2=10000 /$ stocking, which has units of $\mathrm{m}^{2} /$ tree - i.e. the average area available to each tree.

The relationships between the main physiological covariates — $h t, m$, and $d o b 1.3$ - and age 1 were not all the same, as can be seen in Figure 2 Both $h t$ and $d o b 1.3$ increased with $\sqrt{\operatorname{age} 1}$, whilst $m$ grew with $\operatorname{age} 1^{2}$.

[Fig. 2 about here.]

\section{Modelling approach}

We applied nonlinear mixed-effects models to model both the Abies and Eucal datasets. This approach allowed us to look directly at the allometric form of the model, untransformed, and to use the structure of 
the data, namely the repeated measurements on trees in the Abies dataset and the site groupings in the Eucal dataset.

We used only one base functional form, namely the non-linear allometric function, in order to most sharply test the WBE model. Potential deviations from the base form were captured by modeling the fixedeffects parameters $\alpha$ and $\beta$ as smooth functions of predictor variables, such as linear or non-linear functions of tree age. We assessed the utility of both fixed and random effects using whole-model likelihood ratio tests and the visual examination of key plots of functions of residuals, for example auto-correlation. However, comparatively little model-construction testing was required because of the purpose to which the models were being put: our goal was to interpret model short-comings in the context of the failure of the underlying hypothesis as opposed to building a better fitting model.

We considered the use of Reduced Major Axis (RMA) regression as recommended by Warton et al. (2006) and O'Connor et al. (2007) when all variables under consideration are subject to measurement error, but decided it was more important to incorporate the mixed effects given the structure of our data (available RMA software does not easily accommodate mixed effects). See Section 5.3 for further discussion of these modelling assumptions.

A reviewer of an early draft correctly pointed out that the inclusion of age in an allometric modeling exercise is problematic because age relates poorly to size and in any case can be difficult to measure. We agree with these sentiments however in this instance our desire is to assess whether the relationship between tree dimensions changes with maturity, and to then condition upon one of those dimensions, such as diameter, as a proxy for age, introduces an awkward circularity. Furthermore, in these cases we are quite confident that tree ages are measured with sufficient accuracy; the Eucal trees were planted and the Abies trees were split longitudinally and decadal measures taken of stem diameter and terminal bud height taken using tree rings, which are defensibly annual for this species.

\subsection{Nonlinear mixed-effects model}

Our modelling approach borrowed heavily from the methodology in Pinheiro and Bates (2000). For $m$ groups, each with $j=1, \ldots, n_{i}$ observations, the general form of our non-linear mixed-effects model for $i=1, \ldots, m$ can be expressed as:

$$
y_{i j}=f_{\alpha} x_{i j}^{f_{\beta}}+\varepsilon_{i j}
$$

where

$$
f_{\alpha}=X_{\alpha} \gamma_{\alpha}+Z_{\alpha} b_{\alpha} \text { and } f_{\beta}=X_{\beta} \gamma_{\beta}+Z_{\beta} b_{\beta}
$$

also $X_{\alpha}$ and $X_{\beta}$ are design matrices for fixed-effects terms, $\gamma_{\alpha}$ and $\gamma_{\beta}$ are vectors of fixed-effects parameter estimates, $Z_{\alpha}$ and $Z_{\beta}$ are design matrices for random-effects terms, and $b_{\alpha}$ and $b_{\beta}$ are vectors of randomeffects parameter estimates. 
If assumptions of independence and homoscedasticity apply, then we would furthermore have $b_{\alpha} \sim$ $\mathscr{N}\left(0, \sigma_{\alpha}^{2} I\right), b_{\beta} \sim \mathscr{N}\left(0, \sigma_{\beta}^{2} I\right)$, and $\varepsilon_{i} \sim \mathscr{N}\left(0, \sigma^{2} I\right)$, that is, $b_{\alpha}, b_{\beta}$ and the $\varepsilon_{i}$ are assumed to be independent, and the effects and errors of each group are also assumed to be independent of one another. As one or other of these assumptions did not apply in our applications, we also tested for correlated errors and non-constant variance (see online supplement for more details).

\subsection{Testing adequacy of fit of WBE-derived models}

We used two approaches to test the adequacy of fit of our models, namely a regression-based equivalence test proposed in Robinson et al. (2005), and generalised additive models (Wood, 2006). We distinguish here between a model that satisfies its assumptions, so it is internally consistent, e.g., having homoskedastic and normally-distributed errors, and a model that appropriately represents the variation in the data. This section assesses whether the WBE-derived models capture the variation in allometric form expressed within the data.

The regression-based equivalence test looks at the similarity of the model to the data used to fit it at a population- and observation-level, to judge the equivalence of the model and the data. A nice consequence is that any kind of model can be tested in this way, since the test is based only on observations and predictions. The process also automatically incorporates judgments about the practical significance of differences through the specification of 'regions of indifference'. A full description of this equivalence test is provided in the AS supplement.

Generalised additive models are extension of a general linear models where the response is modelled as a sum of smooth functions of the covariates (Hastie and Tibshirani, 1986). For each model fitted, we took the fixed-effects residuals as the GAM response $y_{i}$ and the allometric predictor as the GAM predictor $x_{i}$. We fitted the GAMs using the gam function of the mgcv package in $\mathrm{R}$. We chose the default $k=10$ knots for each, and a penalty for each effective degree of freedom of $\gamma=1.4$ as suggested by Wood (2006, p. 224). The optimal smoothing parameter $\lambda$ was chosen using generalized cross-validation based on UBRE (un-Biased Risk Estimator) within the gam model fitting process.

\subsection{Parameter testing}

Our overarching goal was to assess the veracity of the WBE model for these data. WBE posits particular values for the allometric parameter estimates, so, given a model fit that passed our suite of model assessments, we could interpret the model's parameter estimates in the light of WBE. We performed this assessment by using equivalence tests, which are a variation on traditional tests of statistical significance.

Traditional tests of statistical significance require specification of a null hypothesis, which is commonly that the true value for some estimated parameter is a known constant. The process then either accepts or rejects the null hypothesis (Neyman/Pearson) or reports a measure of the evidence against the null hypothesis (Fisher). This approach is unsatisfactory for the purpose of our study, because we would prefer to specify a 
null hypothesis that the true value for some estimated parameter is not a known constant, and let the method accept or reject the null hypothesis or report a measure of the evidence against the null hypothesis. The latter approach falls under an umbrella of tests called 'equivalence tests' (Parkhurst, 2001, Robinson and Froese. 2004).

Several advantages follow. One is the consequence that the reversed hypothesis has on the type I error rate. We now control the probability of mistakenly validating WBE (previously we controlled the probability of mistakenly rejecting WBE) and so can be more confident in validation of the model of no difference than we can be under traditional significance testing (Robinson and Froese, 2004). We can also be sure that increasing the sample size $n$ will improve the accuracy of the test more reliably than under traditional significance testing (Robinson et al., 2005).

Our basic process for the equivalence tests follows (Wellek, 2003, 3). For testing the estimated exponents of the allometric models fitted, our null hypotheses were expressed as $H_{0}: \theta<\theta_{0}-\varepsilon$, or $\theta>\theta_{0}+\varepsilon$, where $\theta_{0}$ is the WBE predicted value of the relevant exponent. Note that we only consider there to be an effect if the deviation from the predicted value is beyond a 'region of indifference' as specified by the value $\varepsilon$. If the confidence interval generated for the parameter is completely contained within the region of indifference $\left[\theta_{0}-\varepsilon, \theta_{0}+\varepsilon\right]$ then we reject the null hypothesis of difference between the parameter and its hypothesized value. As Schuirmann (1981) pointed out, this is equivalent to the simultaneous rejection of the two separate one-sided null hypotheses $H_{0 a}: \theta<\theta_{0}-\varepsilon$ against $H_{1 a}: \theta \geqslant \theta_{0}+\varepsilon$ and $H_{0 b}: \theta>\theta_{0}+\varepsilon$ against $H_{1 b}: \theta \leqslant$ $\theta_{0}-\varepsilon$. The form of the confidence interval is a two one-sided $(1-\alpha) \%$ confidence interval, as used in the two one-sided test (TOST Westlake, 1981).

\subsection{Implementation}

The emphasis for modelling both datasets was placed on obtaining models where all regression assumptions were met, so that meaningful inference could later be made about the parameter estimates and the overall fit of the allometric model. For both the Abies and Eucal datasets, the modelling method used was nonlinear mixed-effects modelling of the proposed allometric form, using the nlme function from the nlme package (Pinheiro et al., 2016) in R (R Core Team, 2016).

For the Abies dataset we treated each tree as an individual, from a population of trees, providing the random effects. With the models from the Abies dataset we were able to examine static allometry. This dataset offered most complication in terms of the most appropriate modelling of the correlation structure of the errors. A range of models for the correlation structure was tried, and an autoregressive-moving average process with lags $p=1$ and $q=1$ was found to combine the most adequate residuals with autocorrelation decreasing sufficiently quickly with time. Each tree was taken as the individual responsible for the random effect. At the conclusion of modelling, the random effects were examined for each forest and habitat (see Section 3.1 in the AS Supplement), to check whether it might be necessary to include either as an additional random effect. 
For the Eucal dataset we used each site as the source of the individual random effect, from an assumed population of sites. The models from the Eucal dataset were used to examine evolutionary allometry for the four relationships modelled. The Eucal dataset offered a greater challenge in its provision of so many potential covariates. For each of the four relationships modeled, we started with the basic allometric model with the coefficient and exponent being constant. Random effects were tested in both the coefficient and exponent to see where they made the most difference. The covariates were added in manually to coefficient and exponent, beginning with those which appeared to have the clearest effect on the response - usually age 1 and stocking2. The transformation of the covariate age 1 has already been discussed in Section 2.2 Inclusion of species also seemed to generally improve the models, but its location - coefficient or exponent — differed. We found that the inclusion of climate data provided little benefit (the case of mass and diameter was the only one for which inclusion of a climate covariate improved the fit). In all four relationships, the fit was greatly improved by making the exponent a function of covariates. The power variance function also improved the fit in all three relationships, usually with age 1 as the covariate. The random effect for the Eucal dataset was assumed to come from each different site.

At each change to the model the diagnostics were examined to assess how the change affected the regression assumptions. In all, over ten models were fitted to the Eucal dataset for each relationship before arriving at those considered to satisfy all the regression assumptions.

\section{Modelling results}

Modelling results are outlined in this section. Standard plots for testing model assumptions - normality of errors and random effects and lack of pattern in residuals - and plots which guided our decisions on inclusion of random effects for various covariates are available in the AS supplement.

\subsection{The Abies dataset}

Given that only one regression covariate other than diameter was available (Age, or age 1 as actually used), the best model relating height and diameter through the allometric equation described the height of the $i^{t h}$ tree at time $t$ as

$$
\text { height. } m_{i t}=\left(\beta_{0}+b_{0 i}+\beta_{1} \sqrt{\text { age } 1}\right) d b h u b . c m^{\alpha_{0}}+\varepsilon_{i t}
$$

with an autoregressive-moving average process of order $p=1, q=1$ specified for each tree, so the withingroup errors at time $t$ were modelled as:

$$
\varepsilon_{t}=\phi_{1} \varepsilon_{t-1}+\theta_{1} a_{t-1}+a_{t} .
$$

The estimated exponent $\alpha_{0}$ was 1.027 compared to the WBE prediction of $2 / 3$ as further discussed in Section 4.5.2. Other parameter estimates and confidence intervals are in the AS supplement. 


\subsection{The Eucal dataset}

\subsubsection{Height vs. diameter}

Using nonlinear least squares, the model that best described the relationship between height and diameter for the Eucal dataset described the height of the $j^{\text {th }}$ tree of species $k$ in the $i^{\text {th }}$ site as

$$
h t_{i j}=\left(\beta_{0}+\left(\beta_{1}+b_{1 i}\right) \sqrt{a g e 1}+\beta_{2} \text { stocking } 2+x_{k}\right) d o b 1.3^{\left(\alpha_{0}+\alpha_{1} \sqrt{a g e 1}\right)}+\varepsilon_{i j}
$$

with $\operatorname{Var}\left(\varepsilon_{i j}\right)=\sigma^{2} \mid$ age $\left.1_{i j}\right|^{2 \delta}$, in which the exponent of diameter varies with the age 1 covariate.

The estimated exponent for diameter varied over the range $(0.39,0.54)$ reducing with increasing age 1 covariate, compared to the WBE prediction of $2 / 3$, as further discussed in Section 4.5.2. Other parameter estimates and confidence intervals are in the AS Supplement.

\subsubsection{Mass vs. diameter}

Using nonlinear least squares, the model that best described the relationship between mass and diameter for the Eucal dataset described the mass of the $j^{\text {th }}$ tree of species $k$ in the $i^{\text {th }}$ site as

$$
m_{i j}=\left(\beta_{0}+b_{0 i}+\beta_{1} \text { age } 1+\beta_{2} a g e 1^{2}+\beta_{3} \text { stocking } 2\right) d o b 1.3^{\left(\alpha_{0}+\alpha_{1} a g e 1^{2}+x_{k}\right)}+\varepsilon_{i j}
$$

with $\operatorname{Var}\left(\varepsilon_{i j}\right)=\sigma^{2}\left|a g e 1_{i j}\right|^{2 \delta}$, and in which the exponent of diameter varies with age 1 and species.

The estimated exponents for diameter for both species varied over a combined range $(2.07,2.68)$ increasing with the age 1 covariate, only approaching the WBE prediction of $8 / 3$ towards the very top of the age range, as further discussed in Section 4.5.2. Other parameter estimates and confidence intervals are in the AS Supplement.

\subsubsection{Height vs. mass}

Using nonlinear least squares, the best-fitting model to describe the relationship between the height and mass of trees in the Eucal dataset described the height of the $j^{\text {th }}$ tree in the $i^{\text {th }}$ site as

$$
h t_{i j}=\left(\beta_{0}+\left(\beta_{1}+b_{1 i}\right) \sqrt{\text { age } 1}+\beta_{2} \text { stocking } 2+\beta_{3} \operatorname{MinT}\right) m^{\left(\alpha_{0}+\alpha_{1} \sqrt{a g e 1}\right)}+\varepsilon_{i j}
$$

with $\operatorname{Var}\left(\varepsilon_{i j}\right)=\sigma^{2} \mid$ age $\left.1_{i j}\right|^{2 \delta}$, MinT represents the monthly average minimum daily air temperature in degrees Celsius, and in which the exponent of mass varies with age 1 .

The estimated exponent for mass varied over the range $(0.16,0.29)$ reducing with increasing age 1 , compared to the WBE prediction of $1 / 4$, as further discussed in Section 4.5.2. Other parameter estimates and confidence intervals are in the AS Supplement. 


\subsection{Remaining variation in the models}

In Table 2 we summarize the estimated standard deviations of the model responses before and after the models were fitted, the estimated standard deviations of the GAMs fitted to the residuals of each model, the $p$-values of the allometric predictor fitted smooths and the percentage deviance of the residuals they explain.

[Table 2 about here.]

Decreases in the estimated standard deviations of all the responses are apparent. Each of the residual models was statistically significantly different from the null model.

For the Abies model, the cubic spline smooth in diameter fitted to the residuals explained a relatively large amount of variation, and was highly statistically significant. Figure 3 supports this conclusion - there is some pattern being captured by the dbhub.cm fitted smooth.

For the Eucal dataset height-mass model, Figure 4 a) shows a fitted smooth that appears close to straight. This may correspond to the group of small fitted values to the left of standardised residuals vs fitted values plot (for details see the AS website). Some deviance was explained by the fitted smooth (Table 2). More curvature is apparent in panels (b) and (c), which correspond to their much higher and slightly higher percentages of deviance explained (Table 2).

[Fig. 3 about here.]

[Fig. 4 about here.]

\subsection{Regression-based equivalence tests of the fitted models}

From the model fitted to the Abies dataset, we were able to test the static allometry model as defined by Strauss (1993). From the model fitted to the Eucal dataset we tested the evolutionary allometry model. In each case, the region of indifference for the intercept was set at plus or minus $25 \%$ of the mean value of the observations, and the region of indifference for the slope was set at 1 plus or minus 0.25 . This choice is briefly addressed in the Discussion.

\subsubsection{Static allometry}

To test static allometry, the fitted values with random effects for the model Abies.hd were collected for trees of age $[1,2)$ decades, $[2,3)$ decades, $[3,4)$ decades, $[4,5)$ decades, and [5,6) decades. For each age group, the regression-based equivalence test detailed by Robinson et al. (2005) was applied (see the AS Supplement for full details of the test).

The two null hypothesis tested were:

1. That the mean of the predictions was not equivalent to the mean of the observations, i.e., that the predictions were biased; and 
2. That the slope of the regression between the predictions and the observations was not 1, i.e., that the predictions and observations were dissimilar.

A joint significance level of $\alpha=0.05$ was used (so for each individual test $\alpha=0.0253$ ), and 100 bootstrap replicates for each test. The results are summarised in Table 3 The null hypothesis of bias was rejected in all cases, and the null hypothesis of dissimilarity was rejected for all decades apart from [1,2).

[Table 3 about here.]

It appears that the allometric model fitted to the Abies dataset is validated by regression-based equivalence testing as a static allometric model for these trees when in their twenties, their thirties, their forties and their fifties, for our $\pm 0.25 \%$ region of indifference. We repeated these tests at decreasing regions of indifference down to $10 \%$. Whilst the null hypothesis of bias was fairly consistently rejected, smaller regions of indifference for the slope lead to a tendency not to reject the null hypothesis of dissimilarity.

\subsubsection{Evolutionary allometry}

To test the fitted models for satisfactory evolutionary allometry, the fitted values for each relationship modeled from the Eucal dataset were collated, and the regression-based equivalence test applied. The null hypotheses were the same as for the testing of static allometry, with results summarised in Table 3 . Both the null hypothesis of bias and the null hypothesis of dissimilarity were rejected for all models.

\subsection{Estimates of the exponent}

To test the comparability of the parameter estimates of the exponents of the fitted models with those predicted by the WBE model, we used equivalence tests of the kind described by Schuirmann (1981) and Wellek (2003).

In general, the null hypothesis

$$
H_{0}: \text { exponent }<\text { predicted exponent }-\varepsilon \text { or }>\text { predicted exponent }+\varepsilon
$$

was tested against the alternative

$$
H_{1} \text { : predicted exponent }-\varepsilon \leqslant \text { exponent } \leqslant \text { predicted exponent }+\varepsilon \text {. }
$$

Using a significance level of 0.05 , two one-sided $95 \%$ confidence intervals were calculated for the estimated exponent (Schuirmann, 1981; Westlake, 1981). The size of the region of indifference $\varepsilon$ was undefined, so that we could see how large the region of indifference would need to be to reject the null hypothesis of dissimilarity in each case. Rejection of the null hypothesis would only occur if the calculated confidence intervals were completely contained within the region of indifference. The variances of the estimated exponents were calculated according to standard formulae and described fully in the supporting documentation. 


\subsubsection{Parameter estimates from the Abies dataset}

For the model Abies.hd, to test whether the exponent in the relationship between height and diameter was $2 / 3$ as predicted by the WBE model, we used the hypotheses:

$$
\begin{aligned}
& H_{0}: \alpha<2 / 3-\varepsilon \text { or } \alpha>2 / 3+\varepsilon \\
& H_{1}: 2 / 3-\varepsilon \leqslant \alpha \leqslant 2 / 3+\varepsilon .
\end{aligned}
$$

The TOST confidence interval was $(0.980,1.074)$. Since the distance between the furthest point of the TOST confidence interval and the predicted value $2 / 3$ was 0.408 , we would require $\varepsilon=0.408$, or roughly $61 \%$ of the predicted value, to reject the null hypothesis. In addition, the traditional $95 \%$ confidence interval to test the Null Hypothesis that the exponent is $\alpha=2 / 3$ is $(0.97,1.08)$ so we also reject that hypothesis. It seems unlikely that the exponent for our model of the relationship between height and diameter could be $2 / 3$.

\subsubsection{Parameter estimates from the Eucal dataset}

For the Eucal dataset, the equivalence test was a little more involved, since in all cases the exponent was a continuous function of at least one covariate. The essential process, however, was the same. The degrees of freedom were taken from the summary output, as were the variances and covariances of all estimators in the exponents. The variances of the estimated exponents were calculated as shown below. The two onesided $95 \%$ confidence intervals were then calculated, and the region of indifference required to reject the null hypotheses of dissimilarity from the WBE-predicted exponents for all situations were calculated. The full calculations for the estimation of the standard errors and confidence intervals are provided in the AS Supplement.

\section{Height vs. diameter}

For the model Eucal.hd, the hypotheses were

$$
\begin{aligned}
& H_{0}: \alpha_{0}+\alpha_{1} \sqrt{\text { age } 1}<2 / 3-\varepsilon \text { or } \alpha_{0}+\alpha_{1} \sqrt{\text { age } 1}>2 / 3+\varepsilon \\
& H_{1}: 2 / 3-\varepsilon \leqslant \alpha_{0}+\alpha_{1} \sqrt{\text { age } 1} \leqslant 2 / 3+\varepsilon .
\end{aligned}
$$

The exponent $\alpha_{0}+\alpha_{1} \sqrt{\text { age } 1}$ had the two one-sided $95 \%$ confidence interval

$$
\left\{\begin{array}{l}
(0.511,0.565) \text { for the minimum age in decades, } 0.1, \\
(0.363,0.422) \text { for the maximum age in decades 1.2. }
\end{array}\right.
$$

As age in decades increases to its maximum value of 1.2 decades, the estimated exponent and its confidence interval decrease, becoming further away from the predicted value of $2 / 3$. This is plotted for the range of values of age in decades in Figure 5

The greatest distance between the TOST confidence intervals and $2 / 3$ was 0.303 . This means that to reject the null hypothesis at all ages observed, a region of indifference of size $\varepsilon=0.303$ would be required, or approximately $45 \%$ of the predicted value of $2 / 3$. The size of this region of indifference suggests that it is 
unlikely that the exponent of diameter could be $2 / 3$. In addition the traditional $95 \%$ confidence intervals for testing if the exponent is $2 / 3$ are $(0.506,0.570)$ at the minimum age 0.1 and $(0.357,0.427)$ at maximum age 1.2 , so again we reject both of these null hypotheses at the $5 \%$ level.

[Fig. 5 about here.]

\section{Mass vs. diameter}

For the model Eucal.md, the hypotheses were

$$
\begin{aligned}
& H_{0}: \alpha_{0}+\alpha_{1} \text { age } 1^{2}+s p p<8 / 3-\varepsilon \text { or } \alpha_{0}+\alpha_{1} \text { age } 1^{2}+s p p>8 / 3+\varepsilon \\
& H_{1}: 8 / 3-\varepsilon \leqslant \alpha_{0}+\alpha_{1} a g e 1^{2}+s p p \leqslant 8 / 3+\varepsilon
\end{aligned}
$$

For E. globulus the exponent $\alpha_{0}+\alpha_{1}$ age $1^{2}+s p p$ had the two one-sided $95 \%$ confidence interval

$$
\left\{\begin{array}{l}
(2.045,2.178) \text { for the minimum age in decades, } 0.1 \\
(2.551,2.813) \text { for the maximum age in decades, } 1.2
\end{array}\right.
$$

and for $E$. grandis the exponent $\alpha_{0}+\alpha_{1} a g e 1^{2}+s p p$ had the two one-sided $95 \%$ confidence interval

$$
\left\{\begin{array}{l}
(2.005,2.146) \text { for the minimum age in decades, } 0.1 \\
(2.512,2.779) \text { for the maximum age in decades, } 1.2
\end{array}\right.
$$

Plots showing how the exponent changes with age in decades for each species are in Figure 6 The estimated does attain the predicted value as age in decades approaches its maximum observed value.

The greatest distance between these confidence intervals and $8 / 3$ was 0.667 . This means that to reject the null hypothesis for both species at all observed ages, a region of indifference of size $\varepsilon=0.667$ would be required, or approximately $25 \%$ of the predicted value of $8 / 3$. The size of this region of indifference suggests that it may not be unlikely that the exponent of diameter could be $8 / 3$. In addition, we do not reject the traditional $95 \%$ tests of an exponent of $8 / 3$ for ages near to the maximum age of 1.2 for either E. globulus or E. grandis, though we do reject for ages near to the minimum age of 0.1 .

[Fig. 6 about here.]

\section{Height vs. mass}

For the model Eucal.hm, the hypotheses were

$$
\begin{aligned}
& H_{0}: \alpha_{0}+\alpha_{1} \sqrt{\text { age } 1}<1 / 4-\varepsilon \text { or } \alpha_{0}+\alpha_{1} \sqrt{\text { age } 1}>1 / 4+\varepsilon \\
& H_{1}: 1 / 4-\varepsilon \leqslant \alpha_{0}+\alpha_{1} \sqrt{\text { age } 1} \leqslant 1 / 4+\varepsilon
\end{aligned}
$$

The exponent $\alpha_{0}+\alpha_{1} \sqrt{\text { age } 1}$ had the two one-sided $95 \%$ confidence interval

$$
\left\{\begin{array}{l}
(0.273,0.299) \text { for the minimum age in decades, } 0.1 \\
(0.148,0.172) \text { for the maximum age in decades, } 1.2
\end{array}\right.
$$


As with model Eucal.hd, the value of the exponent decreases as age in decades increases. This is shown in Figure 7 .

The greatest distance between these confidence intervals and $1 / 4$ was 0.102 . This means that to reject the null hypothesis at all observed ages, a region of indifference of size $\varepsilon=0.102$ would be required, or approximately $41 \%$ of the predicted value of $1 / 4$. The size of this region of indifference suggests that it is unlikely that the exponent of diameter here could be $1 / 4$.

[Fig. 7 about here.]

\subsubsection{Parameter estimates overview}

Table 4 orders all the fitted models from smallest percentage region of indifference for rejection of the null hypothesis to largest. The results are consistent with the previous observed measures of adequacy of fit for the models.

The largest regions of indifference were needed for model Abies.hd, which had the least satisfactory residuals, the most deviance explained by the GAM, and the poorest performance in the regression-based equivalence test of their fits.

Model Eucal.md required the smallest percentage of the predicted exponent as region of indifference to reject the null hypothesis of dissimilarity from the WBE-predicted exponents. This model also had very good diagnostic plots, the least amount of deviance explained by the GAM, and a very good performance in the regression-based equivalence test of model fit.

Models Eucal.hd and Eucal.hm, which required similar regions of indifference, are not so straightforward. Model Eucal.hd had good diagnostics, had essentially no deviance explained by the GAM, and performed well in the regression-based equivalence test of model fit. Model Eucal.hm had excellent diagnostics, and performed extremely well in the regression-based equivalence test of model fit, but still had some deviance (less than Abies.hd but more than Eucal.hd) explained by the GAM. However, although their percentage regions of indifference are similar, the absolute size of the region of indifference for Eucal.hm is approximately a third that of Eucal.hd.

[Table 4 about here.]

\section{Discussion of results}

\subsection{Accuracy of WBE components}

For the estimated exponents of the four models fitted, the results of the equivalence tests were mixed. Using a significance level of 0.05 , the regions of indifference required to reject the null hypotheses of difference from the WBE predictions were large proportions of the predicted exponent itself (see Table 4). The smallest region of indifference required was $25 \%$ of the value of the predicted exponent, for model Eucal.md. This 
makes it necessary to think carefully about the level of precision we believe should be required to say that there is equivalence between the estimator and the prediction. If we adhere to the 'strict' indifference of $25 \%$ as suggested by Wellek (2003) in the context of the paired t-test for equivalence, then rejection of the null hypotheses and acceptance of the existence of the WBE quarter-power scaling exponents looks doubtful in all cases but that of the relationship between mass and diameter. Examination of Figure 1 shows that the relationship between mass and diameter raised to the WBE-predicted power of $8 / 3$ demonstrates the best correspondence of all the relationships tested from the Eucal dataset. It may not be surprising that this is the only model that seems to come close to supporting the WBE-predicted exponents in formal tests. For the other models, however, quarter-power scaling does not appear to be indicated. A point worth noting with regard to the Eucal models is that the results and conclusions of the interval tests can only be said to apply for relatively young trees. This point is worth noting, as the maximum age observed in the Eucal dataset was 12 years. A test of models of this type fitted to a dataset of trees over a lifetime more comparable to that of the trees from the Abies dataset could provide very different results.

\subsection{Adequacy of Allometric form}

In terms of the adequacy of the general allometric model to describe the relative growth of tree characteristics, the results were also mixed.

The model Abies.hd was used to test the adequacy of the allometric model in terms of static allometry. Using a joint significance level of 0.05 , the regression-based equivalence test required a region of indifference of between $20 \%$ and $25 \%$ of the mean of the observations (for bias) and the slope of 1 (for dissimilarity) to reject both null hypotheses and conclude similarity between the model and the data. This size for the region of indifference was not large enough to conclude similarity for trees aged between 1 and 2 decades. This may indicate that while the model supports static allometry for trees aged between 2 and 6 decades for this region of indifference, there may be a different pattern of growth for young trees that the model is not complex enough to capture.

The allometric model's performance in terms of evolutionary allometry was tested using the three relationships modeled from the Eucal dataset. At a significance level of 0.05 , for all relationships, a region of indifference of only $5 \%$ of the mean of observations and the slope of 1 was sufficient to reject both null hypotheses. The adequacy of the evolutionary allometric model is certainly supported by this data. It must again be noted, however, that this dataset only allowed us to test evolutionary allometry for very young trees, and we cannot say whether or not these results and conclusions might change for tests of trees observed over longer lifetimes.

It also appears from examination of the diagnostic plots of standardized residuals against fitted values (see AS website), and of the GAMs, that there is still some pattern in the relationship between the main allometric response and predictor variables that these allometric models do not capture. Generally this lack of fit seems to occur in the direction of small trees. The most likely source of such variation in the model 
is the existence of a different pattern of growth for young trees and mature trees. Although the difference in biomass production between trees at a stage of life devoted entirely to growth and trees at a stage of life involving reproduction has been suggested by (Enquist. 2002) as a potential cause of such a pattern, it is more likely a result of the slowing in height growth, and the increase in stem girth as trees age. The proportion of annual production allocated to height growth and photosynthetic organs dominates the early stages of tree growth, followed by allocation dominated by development of heartwood (support structure), after which significant growth is allocated to maintenance of the forest through litter fall (Attiwill, 1979). The Eucal dataset is dominated by trees in the height growth phase.

\subsection{Modelling assumptions}

As mentioned in Section 3 , we decided to use a nonlinear modelling procedure based on ordinary least squares methods and including random effects, rather than using an RMA regression. In doing so we assumed that there was little or no measurement error present in any of the predictor variables.

Both ordinary and two-one-sided (TOST) confidence intervals were computed for the parameter estimates using approximate Wald intervals computed from quantities extracted from the non-linear model fits provided by the nlme package. The intervals are approximate in any case, but require the assumption that the underlying data and random effects are conditionally normal, absent large-sample heroics. In general, our assessment of the model diagnostics supported the assumption of conditional normality (see supplementary materials).

We expected that there will have been minimal measurement error in the stem diameter observations for the Abies dataset, but different protocols for biomass measurement across sites in the Eucal dataset may have introduced some measurement error. It is possible that the use of site as the source of random variation may have absorbed some of the variation due to different measurement protocols at different sites, but only very crudely.

According to Hui and Jackson (2007), the presence of this unaccounted-for measurement error may have the effect of increasing the probabilities of the type I and type II errors of any hypothesis tests undertaken on the parameter estimates, particularly of the allometric exponent. This means that in our tests of the exponents in Section 4.5 , we would have had increasing probability of rejecting the null hypothesis of dissimilarity from the WBE-predicted exponents, if the null were true, as any measurement error increased. Comfortingly, there was only one instance in these tests where we would consider rejecting the null hypothesis.

The two dominant model-fitting approaches used in the fitting of the allometric function are the non-linear modeling approach used here, and the linear log-log transformation. Studies have compared the approaches, for example Lai et al. (2013) preferred the linear $\log -\log$ model for coarse root biomass, Mascaro et al. (2011) noted that non-linear regression without further modeling of the residuals introduced substantial bias to the parameter estimates in young forests, and Xiao et al. (2011) suggested that the choice should depend on whether residuals were naturally additive or multiplicative, although see Packard (2014). A comprehensive 
review of the modeling choice is beyond the scope of this paper, however, the literature does not appear to unequivocally support one approach over the other. We elected a mixed-effects non-linear modeling approach with a variance model, so we are confident that the choice will not have affected the outcome of the study.

Hui and Jackson (2007) also claimed that the use of a nonlinear model tends to provide higher estimates of the allometric exponent than with linear log-log transformed models. Since in most cases the estimates of the exponents from our models fell below the predicted exponent (see Figures 55 77, this does not seem to lend much support to the validity of the WBE-predicted exponents.

The fitted models did not support the specific quarter-power exponents predicted by the WBE model for young trees, except in the relationship between mass and diameter. The general allometric model with the exponent as a function of covariates fared better, particularly in the guise of evolutionary allometry. The fitted models strongly supported the evolutionary allometric relationship between height and diameter, mass and diameter, and height and mass for young trees. The fitted models also supported the static allometric relationship. It would also be desirable to test the allometric exponents and evolutionary allometry more thoroughly over a larger range of tree ages if suitable data are available. The most interesting avenue for future work would appear to be the incorporation of different relative growth relationships for different tree ages, to provide a more accurate picture of the relative growth of tree characteristics.

Finally, the use of equivalence tests requires the selection of regions of indifference, $\varepsilon$, as well as the test size. This requirement introduces an uncomfortable illusion of greater subjectivity than the traditional tests, which in any case require specification of the test size. We reported results for arbitrarily selected regions of indifference of $\pm 25 \%$, but performed tests at a range of levels, and reported the results in sufficient detail that the readership can apply their own standards and arrive at their own conclusions (see, e.g., Tables 3 and 4 .

\section{Conclusions}

Our objective was to assess the validity of the allometric model relating morphological characteristics of trees, with particular emphasis on the quarter-power allometric exponents predicted by the WBE model. Fitting allometric models to the relationships between a number of different tree characteristics in two separate datasets allowed us to examine both of these models. In only one of the five models fitted was the existence of the WBE-predicted quarter-power exponent supported. The general allometric model, however, was supported by the models fitted, most emphatically in the case of evolutionary allometry - relating tree characteristics over a population and time. The general allometric model in the context of relating tree dimensions over a population at a single stage of life was supported for trees older than two decades. In all tests carried out, there was a strong indication that these allometric models of relative growth are influenced by the age of the trees in question. 
Acknowledgements The authors are grateful to S. Livesley (the University of Melbourne), and S. Elms (HVP Plantations Pty Ltd) for contributing portions of the analyzed datasets. The clarity of the paper has been greatly improved by the thoughtful comments of two anonymous reviewers.

\section{References}

Attiwill, P. (1979). Nutrient cycling in a Euclayptus obliqua (L'Hérit.) forest. III: Growth, biomass and net primary production. Australian Journal of Botany, 27:439-458.

Coomes, D. A. and Allen, R. B. (2009). Testing the metabolic scaling theory of tree growth. Journal of Ecology, (97):1369-1373.

Coomes, D. A., Lines, E. R., and Allen, R. B. (2011). Moving on from metabolic scaling theory: hierarchical models of tree growth and asymmetric competition for light. Journal of Ecology, (99):748-756.

Enquist, B. J. (2002). Universal scaling in tree \& vascular plant allometry: toward a general quantitative theory linking plant form and function from cells to ecosystems. Tree Physiology, 22:1045-1064.

Enquist, B. J., Charnov, G. B. W. E. L., and Brown, J. H. (1999). Allometric scaling of production and life-history variation in vascular plants. Nature, 401:907-911.

Hastie, T. and Tibshirani, R. (1986). Generalized additive models. Statistical Science, 1(3):297-310.

Hui, D. and Jackson, R. B. (2007). Uncertainty in allometric exponent estimation: A case study in scaling metabolic rate with body mass. Journal of Theoretical Biology, 249:168-177.

Huxley, J. S. (1932). Problems of Relative Growth. Foundations of Natural History. The Johns Hopkins University Press.

Lai, J., Yang, B., Lin, D., Kerkhoff, A. J., and Ma, K. (2013). The allometry of coarse root biomass: Logtransformed linear regression or nonlinear regression? PLOS ONE, 8(10):1-8.

Mäkela, A. and Valentine, H. (2006). Crown ratio influences allometric scaling in trees. Ecology, 87(12):2967-2972.

Mascaro, J., Litton, C. M., Hugehs, R. F., Uowolo, A., and Schnitzer, S. A. (2011). Minimizing bias in biomass allometry: Model selection and log-transformation of data. Biotropica, 43(6):649-653.

Nygren, P. and Pallardy, S. G. (2008). Applying a universal scaling model to vascular allometry in a singlestemmed, monopodially branching deciduous tree (attim's model). Tree Physiology, 28:1-10.

O’Connor, M. P., Agosta, S. J., Hansen, F., Kemp, S. J., Sieg, A. E., McNair, J. N., and Dunham, A. E. (2007). Phylogeny, regression, and the allometry of physiological traits. The American Naturalist, 170:431-442.

Packard, G. C. (2014). On the use of log-transformation versus nonlinear regression for analyzing biological power laws. Biological Journal of the Linnean Society, 113(4):1167-1178.

Parkhurst, D. F. (2001). Statistical significance tests: Equivalence and reverse tests should reduce misinterpretation. Bioscience, 51:1051-1057.

Pinheiro, J., Bates, D., DebRoy, S., Sarkar, D., and R Core Team (2016). nlme: Linear and Nonlinear Mixed Effects Models. R package version 3.1-128. 
Pinheiro, J. C. and Bates, D. M. (2000). Mixed-Effects Models in S and S-PLUS. Statistics and Computing. Springer-Verlag New York, Inc.

R Core Team (2016). R: A Language and Environment for Statistical Computing. R Foundation for Statistical Computing, Vienna, Austria.

Robinson, A. P., Duursma, R. A., and Marshall, J. D. (2005). A regression-based equivalence test for model validation: shifting the burden of proof. Tree Physiology, 25:903-913.

Robinson, A. P. and Froese, R. E. (2004). Model validation using equivalence tests. Ecological Modelling, 176:349-358.

Rüger, N. and Condit, R. (2012). Testing metabolic theory with models of tree growth that include light competition. Functional Ecology, 26:759-765.

Schuirmann, D. L. (1981). On hypothesis testing to determine if the mean of a normal distribution is contained in a known interval. Biometrics, 37:617.

Stage, A. R. (1963). A mathematical approach to polymorphic site index curves for Grand fir. Forest Science, $9(2): 167-180$.

Stark, S. C., Bentley, L. P., and Enquist, B. J. (2011). Response to coomes \& allen (2009) 'testing the metabolic scaling theory of tree growth. Journal of Ecology, (99):741-747.

Strauss, R. E. (1993). Problems of Relative Growth, chapter The Study of Allometry Since Huxley, pages xlvii-lxxv. Foundations of Natural History. The Johns Hopkins University Press.

Warton, D. I., Wright, I. J., Falster, D. S., and Westoby, M. (2006). Bivariate line-fitting methods for allometry. Biological Reviews, 81:259-291.

Wellek, S. (2003). Testing Statistical Hypotheses of Equivalence. Chapman \& Hall/CRC.

West, G. B., Brown, J. H., and Enquist, B. J. (1999). A general model for the structure and allometry of plant vascular systems. Nature, 400:664-667.

West, G. B., Brown, J. H., and Enquist, B. J. (2000). Scaling in Biology, chapter Quarter-Power Allometric Scaling in Vascular Plants: Functional Basis \& Ecological Consequences, pages 167-198. Santa Fe Institute Studies in the Sciences of Complexity. Oxford University Press.

Westlake, W. J. (1981). Response to Thomas B. L. Kirkwood: Bioequivalence testing — a need to rethink. Biometrics, 37:589-594.

Wood, S. N. (2006). Generalized Additive Models An Introduction with R. Texts in Statistical Science. Chapman \& Hall/CRC.

Xiao, X., White, E. P., Hooten, M. B., and Durham, S. L. (2011). On the use of log-transformation vs. nonlinear regression for analyzing biological power laws. Ecology, (92):1887-1894. 


\section{List of Figures}

1 Model responses against WBE-transformed predictors for Eucal dataset - the plot of height and diameter raised to the power of $2 / 3$ (a) does not suggest a constant linear association. Small and large trees appear to follow a different relationship than those in the middle. The relationship between mass and diameter raised to the power of $8 / 3$ (b) demonstrates an excellent one-to-one correspondence, suggesting that this relationship may well be proportional. The relationship between height and mass raised to the power of 1/4 (c) appears quite linear for trees above $5 \mathrm{~m}$, but for trees below this height a different pattern is visible. $\ldots . . . .23$

2 Relationships between the main morphological covariates for Eucal dataset - height (a), diameter (b), and mass (c) against transformed age in decades with loess fitted smooth (in red, with smoothing parameter 2/3). The transformations of age 1 are those that provide the most linear relationship between the $y$ - and $x$-axis covariates. $\ldots \ldots \ldots . \ldots 24$

3 Cubic spline fitted smooth applied to the residuals of the model Abies.hd - the dbhub.cm fitted smooth is clearly capturing some variation remaining in the model. . . . . . . . . . 25

4 Cubic spline fitted smooths applied to the residuals of the models from the Eucal dataset -

\begin{tabular}{|l}
\hline the height-diameter (a) and mass-diameter (b) models show residual shape, and the mass- \\
diameter graph shows evidence of stratification. For the height-mass model (c), the fitted
\end{tabular} smooth in mass appears to be capturing a little pattern in the far left. . . . . . . . . . . . 26

5 Equivalence plot for test of exponent for model Eucal.hd (height predicted by diameter) the black line shows the estimated value of the exponent, decreasing as age in decades increases and surrounded by its two one-sided $95 \%$ confidence interval (dashed black lines). The WBE-predicted exponent of $2 / 3$ is shown by the solid blue line. The dashed blue lines demonstrate the size of the region of indifference necessary to reject the null hypothesis at all ages obesrved. . . . . . . . . . . . . . . . . . . . 27

6 Equivalence plot for test of exponent for model Eucal.md (mass predicted by diameter) the black line shows the estimated value of the exponent, increasing with age in decades and surrounded by its two one-sided 95\% confidence interval (dashed black lines). The WBEpredicted exponent of $8 / 3$ is shown by the solid blue line. The dashed blue lines demonstrate the size of the region of indifference necessary to reject the null hypothesis at all ages observed. The estimate of the exponent and the necessary region of indifference are slightly larger for E. grandis than for E. globulus. $\ldots \ldots \ldots \ldots \ldots$

7 Equivalence plot for test of exponent for model Eucal.hm (height predicted by mass) the black line shows the estimated value of the exponent, decreasing as age in decades increases and surrounded by its two one-sided 95\% confidence interval (dashed black lines). The WBE-predicted exponent of $1 / 4$ is shown by the solid blue line. The dashed blue lines demonstrate the size of the region of indifference necessary to reject the null hypothesis at

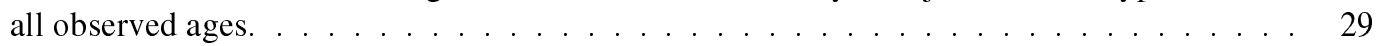




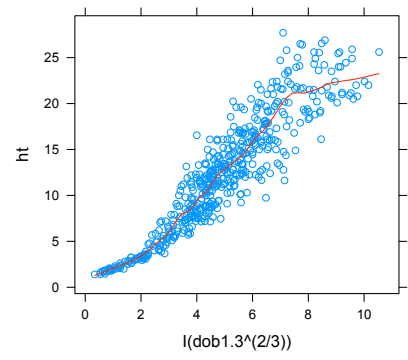

(a) $h t$ vs. $d o b 1.3^{2 / 3}$

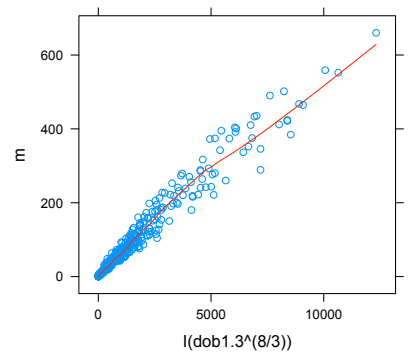

(b) $m$ vs. $d o b 1.3^{8 / 3}$

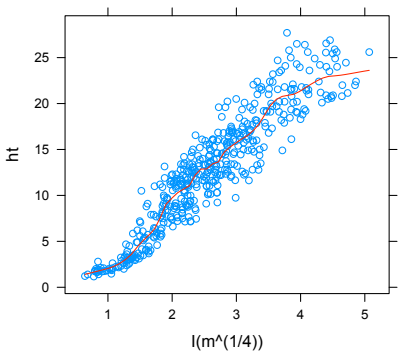

(c) $h t$ vs $m^{1 / 4}$

Fig. 1 Model responses against WBE-transformed predictors for Eucal dataset - the plot of height and diameter raised to the power of 2/3 (a) does not suggest a constant linear association. Small and large trees appear to follow a different relationship than those in the middle. The relationship between mass and diameter raised to the power of $8 / 3$ (b) demonstrates an excellent one-to-one correspondence, suggesting that this relationship may well be proportional. The relationship between height and mass raised to the power of $1 / 4$ (c) appears quite linear for trees above $5 \mathrm{~m}$, but for trees below this height a different pattern is visible. 


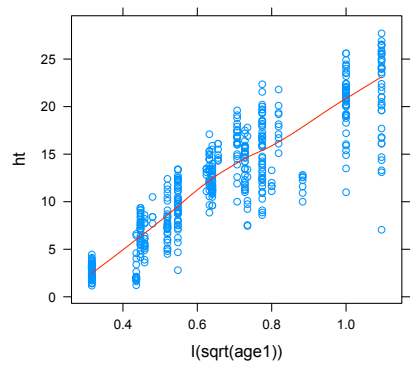

(a) $h t$ against $\sqrt{\text { age } 1}$

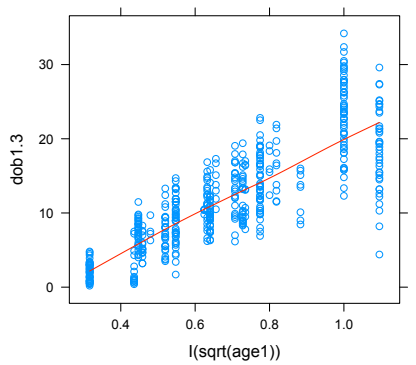

(b) dob1.3 against $\sqrt{\text { age } 1}$

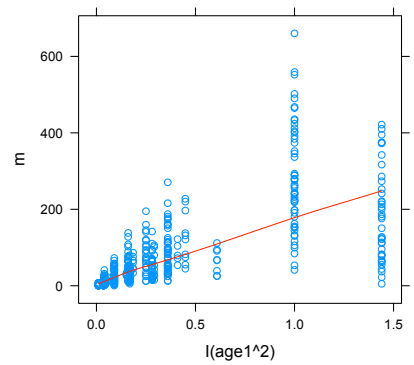

(c) $m$ against age $1^{2}$

Fig. 2 Relationships between the main morphological covariates for Eucal dataset - height (a), diameter (b), and mass (c) against transformed age in decades with loess fitted smooth (in red, with smoothing parameter 2/3). The transformations of age 1 are those that provide the most linear relationship between the $y$ - and $x$-axis covariates. 


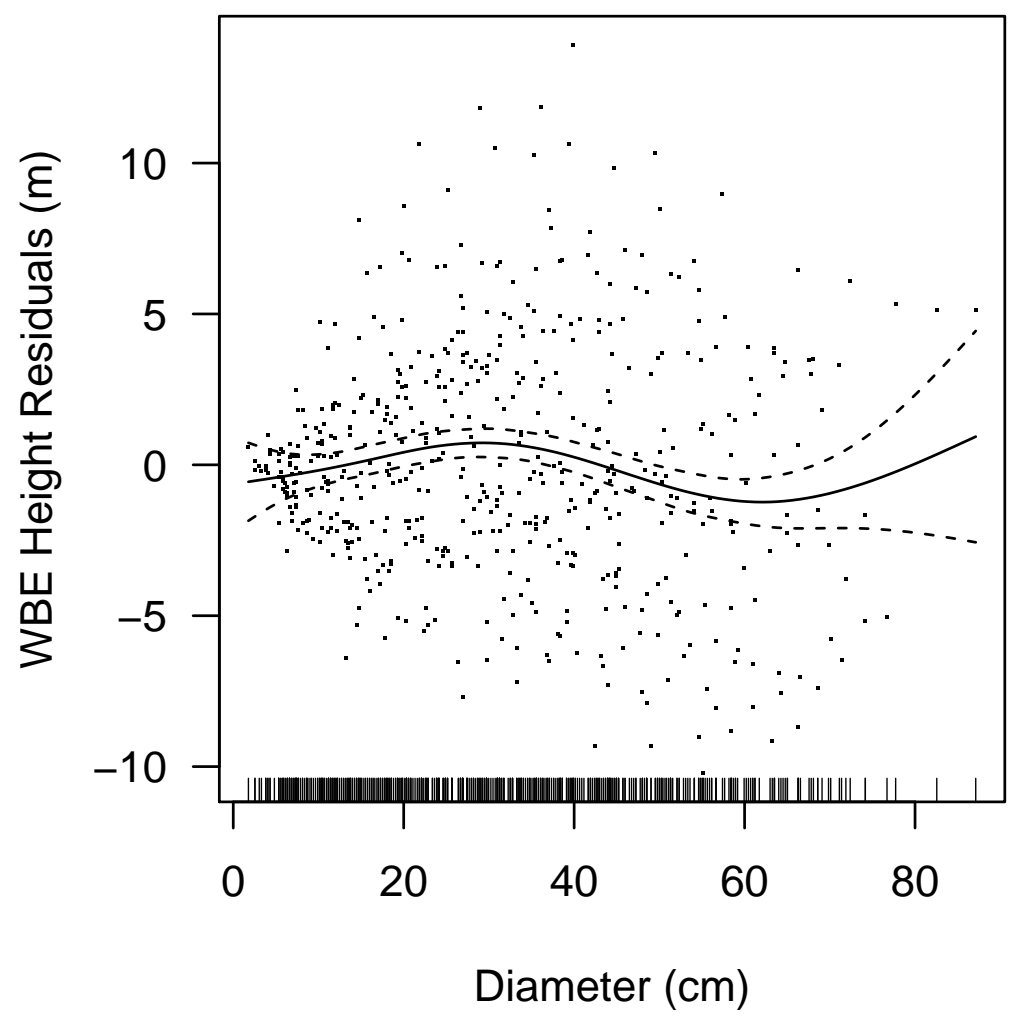

Fig. 3 Cubic spline fitted smooth applied to the residuals of the model Abies.hd - the dbhub.cm fitted smooth is clearly capturing some variation remaining in the model. 

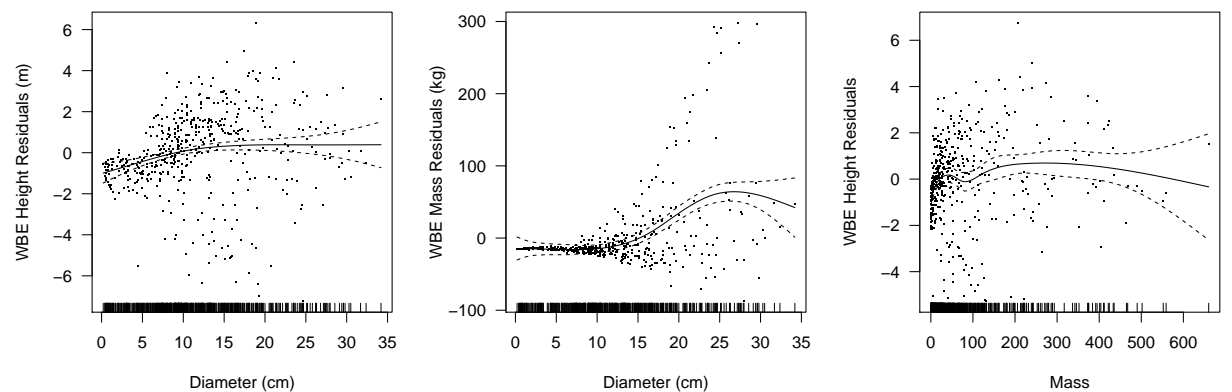

(a) Diameter fitted smooth for model (b) Diameter fitted smooth for model (c) Mass fitted smooth for model EuEucal.hd

Eucal.md

cal.hm

Fig. 4 Cubic spline fitted smooths applied to the residuals of the models from the Eucal dataset - the height-diameter (a) and massdiameter (b) models show residual shape, and the mass-diameter graph shows evidence of stratification. For the height-mass model (c), the fitted smooth in mass appears to be capturing a little pattern in the far left. 


\section{Exponent varying with age (decades)}

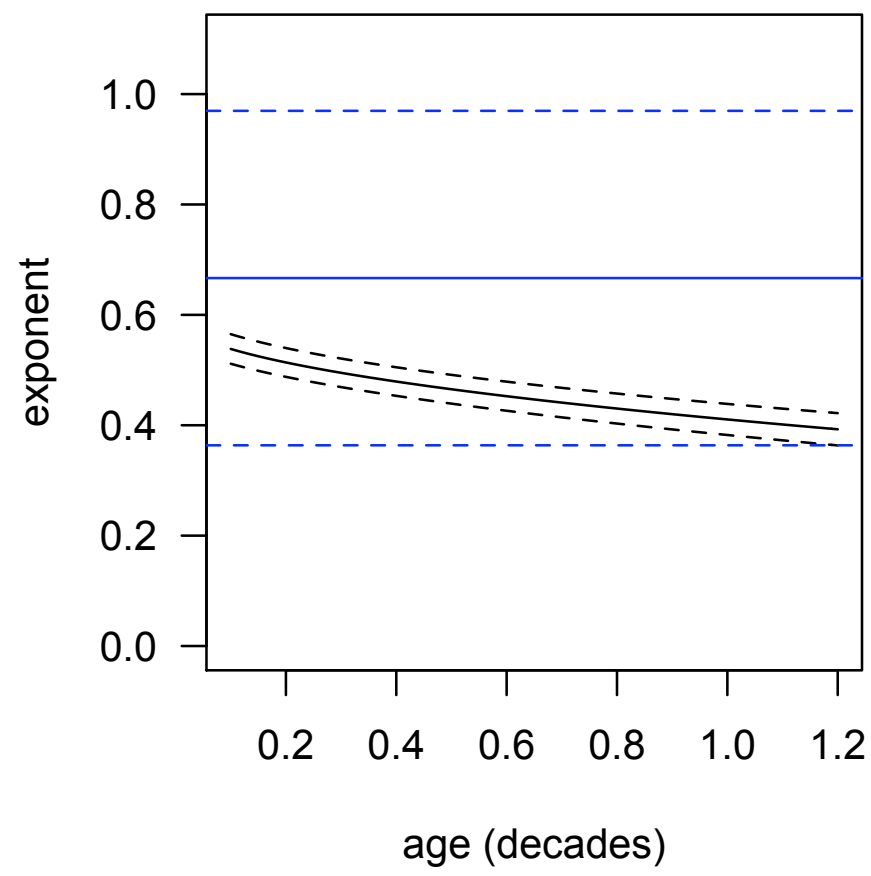

Fig. 5 Equivalence plot for test of exponent for model Eucal.hd (height predicted by diameter) - the black line shows the estimated value of the exponent, decreasing as age in decades increases and surrounded by its two one-sided $95 \%$ confidence interval (dashed black lines). The WBE-predicted exponent of $2 / 3$ is shown by the solid blue line. The dashed blue lines demonstrate the size of the region of indifference necessary to reject the null hypothesis at all ages obesrved. 

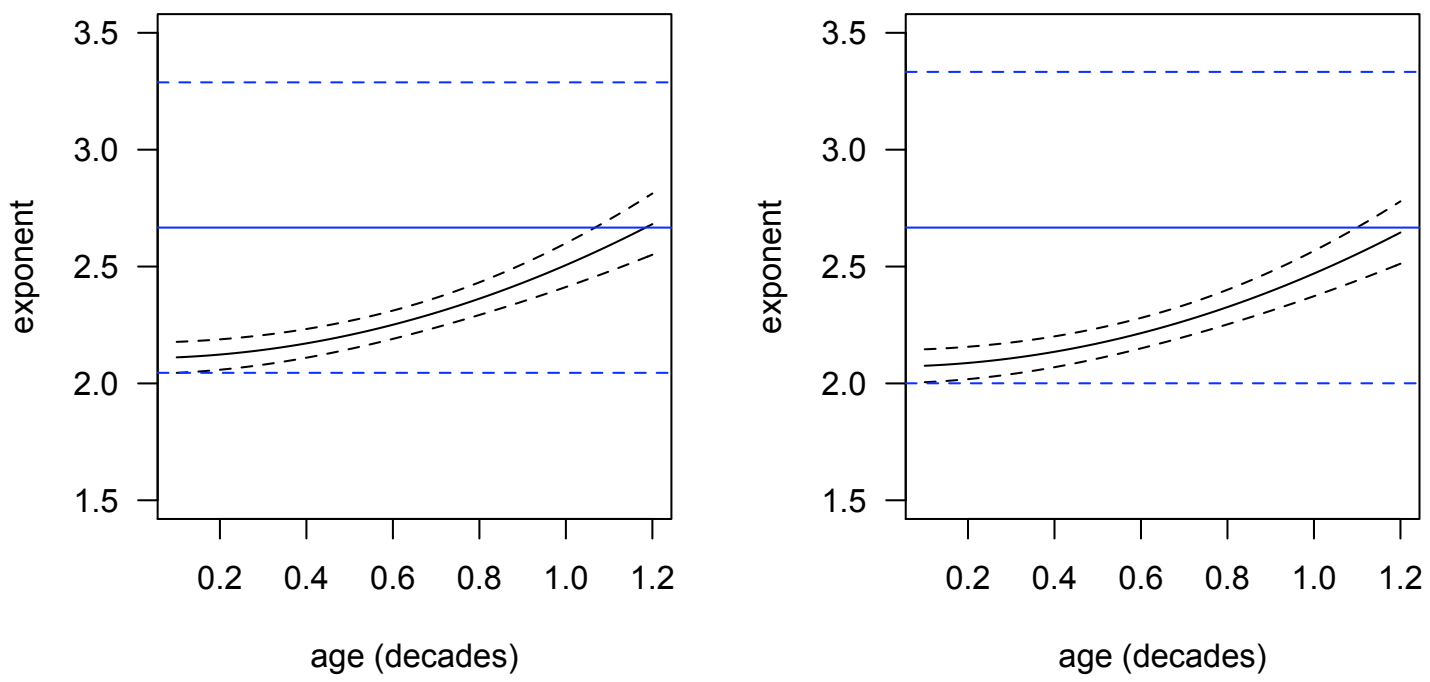

Fig. 6 Equivalence plot for test of exponent for model Eucal.md (mass predicted by diameter) - the black line shows the estimated value of the exponent, increasing with age in decades and surrounded by its two one-sided 95\% confidence interval (dashed black lines). The WBE-predicted exponent of $8 / 3$ is shown by the solid blue line. The dashed blue lines demonstrate the size of the region of indifference necessary to reject the null hypothesis at all ages observed. The estimate of the exponent and the necessary region of indifference are slightly larger for E. grandis than for E. globulus. 


\section{Exponent varying with age (decades)}

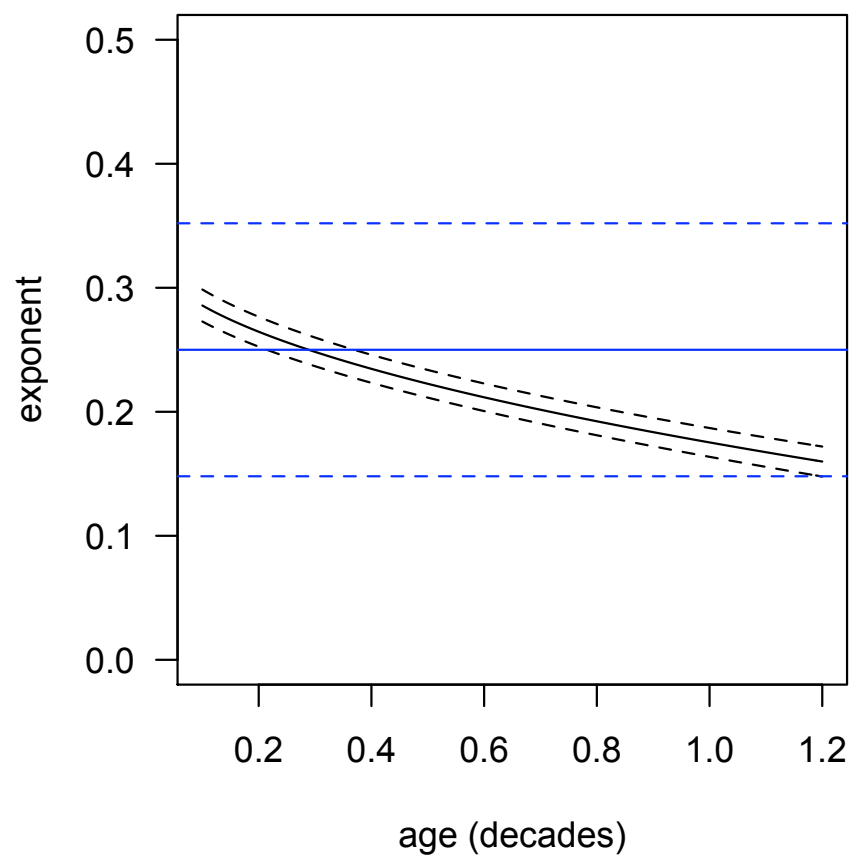

Fig. 7 Equivalence plot for test of exponent for model Eucal.hm (height predicted by mass) — the black line shows the estimated value of the exponent, decreasing as age in decades increases and surrounded by its two one-sided 95\% confidence interval (dashed black lines). The WBE-predicted exponent of $1 / 4$ is shown by the solid blue line. The dashed blue lines demonstrate the size of the region of indifference necessary to reject the null hypothesis at all observed ages. 


\section{List of Tables}

$1 \quad$ A summary of scaling exponents of $M$ for various characteristics predicted by the WBE model, taken from Enquist (2002). . . . . . . . . . . . . . . . 31

2 Estimated standard deviations of response variables, residuals, and residual variation of GAM$\mathrm{s}$ fitted to the residuals of each model, $P$-values of the allometric predictor fitted smooths and the percentage deviance of the residuals that they explain. $\ldots \ldots \ldots 32$

3 Outcomes of the regression-based equivalence test for $25 \%$ regions of indifference for static and evolutionary allometry. Bootstrap intervals for intercept and slope (BS-Intercept and BS-slope) are compared with the corresponding regions of indifference (RI-Intercept and

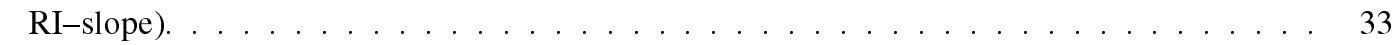

4 Required regions of indifference for the exponents of each model in order to reject the null hypothesis of dissimilarity from the WBE predictions. The models are ordered from smallest required percentage region of indifference to largest. 
Table 1 A summary of scaling exponents of $M$ for various characteristics predicted by the WBE model, taken from Enquist (2002).

\begin{tabular}{lc}
\hline Characteristic & Predicted exponent of $M, \alpha$ \\
\hline No. leaves (Leaf count) & $3 / 4$ \\
No. branches & $3 / 4$ \\
No. tubes & $3 / 4$ \\
Branch length & $1 / 4$ \\
Branch radius & $3 / 8$ \\
Area of conducting tissue & $7 / 8$ \\
Tube radius & $1 / 6$ \\
Conductivity & 1 \\
Leaf-specific conductivity & $1 / 4$ \\
Fluid flow rate & $3 / 4$ \\
Metabolic rate & $3 / 4$ \\
Pressure gradient & $-1 / 4$ \\
Fluid velocity & $-1 / 8$ \\
Branch resistance & $-3 / 4$ \\
Tree height & $1 / 4$ \\
Reproductive biomass & $3 / 4$ \\
Fluid volume & $25 / 24$ \\
\hline
\end{tabular}


Table 2 Estimated standard deviations of response variables, residuals, and residual variation of GAMs fitted to the residuals of each model, $P$-values of the allometric predictor fitted smooths and the percentage deviance of the residuals that they explain.

\begin{tabular}{l|cccc}
\hline Model & Abies.hd & Eucal.hd & Eucal.md & Eucal.hm \\
\hline$s d$ (response) & 12.601 & 6.314 & 109.633 & 6.341 \\
$s d$ (WBE residual) & 3.915 & 2.466 & 53.820 & 2.096 \\
$s d($ GAM residual) & 3.833 & 1.96 & 44.424 & 1.77 \\
$P$-value of GAM fitted smooth & 0.0067 & 0.0001 & $<0.00001$ & 0.00075 \\
Deviance explained by GAM $(\%)$ & $3.39 \%$ & $4.59 \%$ & $24.25 \%$ & $5.41 \%$ \\
\hline
\end{tabular}


Table 3 Outcomes of the regression-based equivalence test for $25 \%$ regions of indifference for static and evolutionary allometry. Bootstrap intervals for intercept and slope (BS-Intercept and BS-slope) are compared with the corresponding regions of indifference (RI-Intercept and RI-slope).

\begin{tabular}{c|cccc}
\hline & BS-Intercept & RI-Intercept & BS-slope & RI-slope \\
\hline Static-Decade & & & & \\
\hline$[1,2)$ & $(5.64,6.28)$ & $(4.66,7.77)$ & $(0.68,1.08)$ & $(0.75,1.25)$ \\
{$[2,3)$} & $(10.00,10.94)$ & $(7.79,12.99)$ & $(0.80,1.09)$ & $(0.75,1.25)$ \\
{$[3,4)$} & $(14.70,15.88)$ & $(11.07,18.44)$ & $(0.90,1.12)$ & $(0.75,1.25)$ \\
{$[4,5)$} & $(19.41,20.91)$ & $(14.60,24.33)$ & $(0.93,1.17)$ & $(0.75,1.25)$ \\
{$[5,6)$} & $(23.18,24.69)$ & $(17.40,29.01)$ & $(0.91,1.14)$ & $(0.75,1.25)$ \\
Evolutionary - Model & & & & \\
\hline Hgt vs. Diameter & $(12.27,12.46)$ & $(9.28,15.47)$ & $(0.98,1.01)$ & $(0.75,1.25)$ \\
Mass vs. Diameter & $(81.15,84.28)$ & $(61.75,102.92)$ & $(0.97,1.03)$ & $(0.75,1.25)$ \\
Hgt vs. Mass & $(12.50,12.70)$ & $(9.46,15.77)$ & $(0.99,1.01)$ & $(0.75,1.25)$ \\
\hline
\end{tabular}


Table 4 Required regions of indifference for the exponents of each model in order to reject the null hypothesis of dissimilarity from the WBE predictions. The models are ordered from smallest required percentage region of indifference to largest.

\begin{tabular}{cccc}
\hline Model & Relationship & Predicted $\alpha$ & Region of indifference to reject $H_{0}$ \\
\hline Eucal.md & $m=\beta d o b 1.3^{\alpha}$ & $8 / 3$ & $25 \%$ of $8 / 3(0.667)$ \\
Eucal.hm & $h t=\beta m^{\alpha}$ & $1 / 4$ & $41 \%$ of $1 / 4(0.102)$ \\
Eucal.hd & $h t=\beta$ dob $1.3^{\alpha}$ & $2 / 3$ & $45 \%$ of $2 / 3(0.303)$ \\
Abies.hd & height. $m=\beta d b h u b . c m^{\alpha}$ & $2 / 3$ & $61 \%$ of $2 / 3(0.408)$ \\
\hline
\end{tabular}


Journal of Agricultural, Biological and Environmental Statistics

\section{The allometric quarter-power scaling model and its applicability to Grand fir and Eucalyptus trees}

Allometric Scaling Supplement

March 2017 


\section{The data}

We used two datasets to critique the WBE model. The first, the Abies dataset, provided height and stem diameter at breast-height (1.37 m, called dbh in Australia), measurements of trees in varied environmental conditions over time (Stage, 1963).

5 The model fitted to this dataset was used to examine ontogenetic allometry and static allometry. The second, the Eucal dataset, provided measurements of such variables as height, breast-height (1.30 m in Australia, $1.37 \mathrm{~m}$ in USA) diameter, mass, and leaf area, for 492 individual trees. The models fitted to this dataset were used to examine evolutionary allometry for a number of different relationships.

\section{1.1 The Abies dataset}

The Abies dataset is a set of measurements of height and breast-height diameter made on 66 grand fir trees growing in various locations and habitats over many decades. The trees were purposively sampled for quality and lack of suppression from nine national forests in the US: Kaniksu, Coeur d'Alene, St. Joe, Clearwater, Nez Perce,

15 Clark Fork, Umatilla, Wallowa and Payette. They grew in five different habitats, as characterised by the 'climax tree species' (the most shade-tolerant species that could grow on a particular tree's site) and the 'dominant understory plant'. The species involved were recorded in abbreviated form:

Th $\mid$ Thuja plicata (Western red cedar)

Ts T. plicata and Tsuga heterophylla (Western hemlock)

AG Abies grandis (Grand fir, also called Lowland fir)

PA Picea engelmanii (Engelmann spruce) and A. lasiocarpa (Subalpine fir)

Pach Pachistima myrsinites (Oregon boxleaf, also called Falsebox)

Op Oplopanaz horridum (Devil's club)

The five habitats (climax tree/dominant understory plant combinations) were 'Ts/Pac', 'Ts/Op', 'Th/Pach', 'AG/Pach', 'PA/Pach'.

The covariates available to us from the Abies dataset are:

- Tree.ID - an identifying number for each tree;

- Forest - an identifying number for each forest;

- HabType - an identifying number for each habitat type;

- Decade - an integer increasing from 0 (representing 1963) to indicate the decade of the measurement;

- Dbhub - the diameter at breast height of the tree, in inches;

- Height - the height of the tree, in feet; 
- Age - the age of the tree, in years.

We included additional variables:

- Forest.ID - each forest's name;

- Hab.ID - each habitat type's name;

- dbhub.cm - the diameter at breast height of the tree, in cm;

- height.m - the height of the tree, in m;

- age 1 - the age of the tree in decades.

\subsection{The Eucal dataset}

40 In this dataset there are measurements made on 492 trees, growing in 19 different sites over southern Australia. These are Shepparton, provided by T. Baker (The University of Melbourne), Napoleons 1 and 2, Tahara and Ullina, provided by S. Livesley (The University of Melbourne), Longford, Toongabbie and Traralgon, provided by C. Weston (the University of Melbourne), Bolivar and Mount Gambier, provided by

45 D. McGuire (Forestry SA), Boyup Brook, Scotts River and Wellstead, provided by I. Dumbrell (Forest Products Commission Western Australia), and Darlimurla, Gormandale, Jeeralang, Moe South, Stradbroke and Yallourn North, provided by S. Elms (HVP Plantations Pty Ltd). We called this the Eucal dataset.

Within each site, trees were grown in a number of stands; within each stand 50 were a number of plots, reflecting a range of treatments, including irrigation and fertilization. Measurements specific to each stand were obtained by averaging over plot measurements.

This data set provided a range of physiological measurements for these trees, a complete list of which is as follows:

- Site - the site in which the tree grew;

- Spp — the species of the tree;

- Stocking (trees/ha) - the planting density of the stand in which the tree grew;

- Age (years) - the age of the tree at the time of its sampling;

- $\operatorname{VOB}\left(\mathrm{m}^{3}\right)$ - total stem volume over bark (i.e., volume of the trunk);

- $\operatorname{VUB}\left(\mathrm{m}^{3}\right)$ - total stem volume under bark;

- LJ sla $\left(\mathrm{m}^{2} / \mathrm{kg}\right)$ - juvenile specific leaf area;

- LA sla $\left(\mathrm{m}^{2} / \mathrm{kg}\right)$ - adult specific leaf area;

- LT sla $\left(\mathrm{m}^{2} / \mathrm{kg}\right)$ - total specific leaf area;

- LJ la $\left(\mathrm{m}^{2}\right)$ - juvenile leaf area; 
- LA la $\left(\mathrm{m}^{2}\right)$ - adult leaf area;

- LT la $\left(\mathrm{m}^{2}\right)$ - total leaf area;

- LJ dm (kg) - juvenile dry mass (leaves);

- LA dm (kg) — adult dry mass (leaves);

- LT dm (kg) - total dry mass (leaves);

- BL dm (kg) — live dry mass (branches);

- BD dm (kg) — dead dry mass (branches);

- BT dm (kg) - total dry mass (branches);

- L_Crown dm (kg) — live crown dry mass;

- T_Crown dm $(\mathrm{kg})$ - dead crown dry mass;

- SB dm (kg) — stem bark dry mass;

- SH dm (kg) — stem heartwood dry mass;

- SS dm (kg) — stem sapwood dry mass;

- SW dm (kg) - stem wood dry mass;

- SW bd $\left(\mathrm{kg} / \mathrm{m}^{3}\right)$ - stem wood basic density;

- SB\&W dm (kg) — stem bark and wood dry mass;

- Total Abg dm (kg) - total aboveground dry mass;

- \% dm foliage - percentage of tree dry mass that is foliage;

- \% dm branches - percentage of tree dry mass that is branches;

- \% dm stem + bark - percentage of tree dry mass that is stem and bark;

- DOB1.3 $(\mathrm{cm})$ - tree diameter over bark at 1.3m height;

- DUB1.3 $(\mathrm{cm})$ - tree diameter under bark at 1.3m height;

- HT (m) - total tree height;

- HG (m) - height of tree up to base of green crown;

- CL (m) - crown length;

- DOB0.1 $(\mathrm{cm})$ - tree diameter over bark at $0.1 \mathrm{~m}$ height;

- DUB0.1 $(\mathrm{cm})$ - tree diameter under bark at 0.1m height;

- DOBG $(\mathrm{cm})$ - tree diameter over bark at base of green crown;

- DUBG $(\mathrm{cm})$ - tree diameter under bark at base of green crown;

- HA0.1 $\left(\mathrm{cm}^{2}\right)$ - heartwood area at $0.1 \mathrm{~m}$ height;

- SA0.1 $\left(\mathrm{cm}^{2}\right)$ - sapwood area at $0.1 \mathrm{~m}$ height;

- HA1.3 $\left(\mathrm{cm}^{2}\right)$ - heartwood area at $1.3 \mathrm{~m}$ height;

- SA1.3 $\left(\mathrm{cm}^{2}\right)$ - sapwood area at $1.3 \mathrm{~m}$ height;

- HAG $\left(\mathrm{cm}^{2}\right)$ - heartwood area at base of green crown; 
- SAG $\left(\mathrm{cm}^{2}\right)$ - sapwood area at base of green crown;

- Thinning - an indicator $(0=$ No, $1=$ Yes $)$ of whether the tree grew in a stand that was subject to thinning;

- Pruning - an indicator $(0=$ No, $1=$ Yes $)$ of whether the tree was pruned;

- Fertilizer - an indicator $(0=$ No, $1=$ Yes $)$ of whether the tree was fertilized;

- Herbicide - an indicator $(0=$ No, $1=$ Yes $)$ of whether or not the tree was treated with herbicide;

- Insecticide - an indicator $(0=$ No, $1=$ Yes $)$ of whether or not the tree was treated with insecticide;

- Irrigation - an indicator $(0=$ No, $1=$ Yes $)$ of whether or not the tree was irrigated.

110 In addition, for each site (and hence also approximately for each tree) we have measurements for:

- $\operatorname{MaxT}\left({ }^{\circ} \mathrm{C}\right)$ - maximum monthly temperature;

- $\operatorname{MinT}\left({ }^{\circ} \mathrm{C}\right)$ - minimum monthly temperature;

- Rain (mm) - total average yearly rainfall;

- Evap (mm) - total average yearly evaporation;

- $\operatorname{Rad}\left(\mathrm{MJ} . \mathrm{m}^{-2}\right.$.day) - total average yearly radiation;

- Lat $\left(^{\circ}\right)$ - latitude of the site;

- Long $\left(^{\circ}\right)$ - longitude of the site;

- Alt $(\mathrm{m})$ — altitude of the site above sea level.

Note that the Eucal dataset available on the JABES website only includes the variables actually used in our analysis.

Several transformations were made to the variables involved. The age of each tree in years was provided, but we used age $1=0.1 \times$ age to enable an interpretation in terms of change in dimension by decade instead of year. The variable stocking

125 was provided in units of trees/hectare. A rearrangement that enabled discussion of the variable in terms more immediately relevant to the individual trees and sites was stocking $2=10000 /$ stocking, which has units of $\mathrm{m}^{2} /$ tree - i.e. the average area available to each tree. 


\section{Detailed modelling results}

\subsection{The Abies dataset}

The Abies dataset had only one covariate available other than diameter (namely age in decades, age1). A range of models for the correlation structure was tried, and an autoregressive-moving average process with lags $p=1$ and $q=1$ was found

135 to combine the most adequate residuals with autocorrelation decreasing sufficiently quickly with time.

Given that only one regression covariate other than diameter was available (Age, or age 1 as actually used), the best model relating height and diameter through the allometric equation described the height of the $i^{t h}$ tree at time $t$ as

$$
\text { height. } m_{i t}=\left(\beta_{0}+b_{0 i}+\beta_{1} \sqrt{a g e 1}\right) d b h u b . c m^{\alpha_{0}}+\epsilon_{i t}
$$

with an autoregressive-moving average process of order $p=1, q=1$ specified for each tree, so the within-group errors at time $t$ were modeled as:

$$
\epsilon_{t}=\phi_{1} \epsilon_{t-1}+\theta_{1} a_{t-1}+a_{t}
$$

Table 2.1: Parameter estimates and confidence intervals for model abies.2.4

\begin{tabular}{|c|c|c|}
\hline Parameter & Estimate & Approx. 95\% C.I. \\
\hline$\beta_{0}$ & 0.850 & $(0.717,0.983)$ \\
$\sigma_{b_{0 i}}$ & 0.066 & $(0.042,0.102)$ \\
$\beta_{1}$ & -0.071 & $(-0.089,-0.052)$ \\
$\alpha_{0}$ & 1.027 & $(0.971,1.084)$ \\
$\sigma$ & 2.552 & $(2.169,3.003)$ \\
$\phi_{1}$ & 0.780 & $(0.698,0.841)$ \\
$\theta_{1}$ & 0.315 & $(0.211,0.411)$ \\
\hline
\end{tabular}

The errors are correlated according to an $\operatorname{ARMA}(1,1)$ process, according to

$$
\hat{\epsilon}_{t}=0.780 \epsilon_{t-1}+0.315 a_{t-1}+a_{t}
$$

and

$$
h(k, \boldsymbol{\rho})=\left\{\begin{array}{l}
0.780 h(|k-1|, \boldsymbol{\rho})+0.315 \psi(|k-1|, \boldsymbol{\rho}), k=1 \\
0.780 h(|k-1|, \boldsymbol{\rho}), k=2,3, \ldots
\end{array}\right.
$$




\subsection{The Eucal dataset}

For each model proposed and fit, attention was paid to the regression assumptions un-

140 derlying each model; the assumption of independence and normality of the residuals, and of the normality of the random effects in the model.

We began with $D O B 1.3$ as the only predictor in the allometric model. At each step, variance structures varIdent, varPower and varConstPower were tried, to see which variance structure would best help with the assumption of normal and indepen-

145 dent residuals. The best-performing at all steps were varPower and varConstPower, although in all but one case the constant was estimated to be near enough to zero as to make no difference.

At each step one other variable was added into the model as a fixed effect, to try to improve on the fit (which, for only having DOB1.3 in the model, was rather

150 dismal). Anova comparisons were used at each step to assess the usefulness of each new variable. By this process $A g e 1, A g e 1^{2}$, Stock2 and Spp were all added into the model in the coefficient of $D O B 1.3$ (computational issues prevented us from testing fixed effects in the exponent of $D O B 1.3)$. The differences in predictive accuracy and indeed in appearances of the residuals is slight many models. The best performing

155 model was chosen by its combination of satisfactory standardized residuals, normality of residuals, approximate normality of the random effect (the coefficient of Age1 within the coefficient of $D O B 1.3)$ and statistical significance of the change in log likelihood between models (a whole-model test).

\subsubsection{Candidate Models}

160 The following list shows the initial suite of height-diameter models compared on the Eucal dataset. Similar sets of models were compared for the mass-diameter and height-mass modeling exercises. These models were also tested with the square root of age, and subsequently predictors were successfully added to the exponent.

- euc.hd.2: $h_{i j}=\left(\beta_{0}+b_{0 i}\right) d_{i j}^{\alpha_{0}}+e_{i j}$,

165 where $\beta_{0}$ is the fixed coefficient, $b_{0 i}$ is the random effect of Site on the coefficient, and $\alpha_{0}$ is the fixed exponent of $d$. We assume that (1) the $e_{i j} \sim$ i.i.d. $\mathcal{N}\left(0, \sigma^{2}\right)$, and (2) the $b_{0 i} \sim$ i.i.d. $\mathcal{N}\left(0, \sigma_{b_{0}}^{2}\right)$.

- euc.hd.3: as euc.hd.2, but that each Site has its own residual variance.

- euc.hd.4: $h_{i j}=\left(\beta_{0}+b_{0 i}+\beta_{1}\right.$ Age $) d_{i j}^{\alpha_{0}}+e_{i j}$,

170 here $\beta_{1}$ represents the fixed effect of Age 1 in the coefficient, and each Site has its own residual variance.

- euc.hd.5: as euc.hd.4, but common variance amongst sites. 
- euc.hd.6: $h_{i j}=\left(\beta_{0}+b_{0 i}+\beta_{1}\right.$ Age $\left.1+\beta_{2} A g e 1^{2}\right) d_{i j}^{\alpha_{0}}+e_{i j}$,

we now have $\beta_{2}$ as the coefficient of $A g e 1^{2}$ in the coefficient.

- euc.hd.7: $h_{i j}=\left(\beta_{0}+b_{0 i}+\beta_{1}\right.$ Age $\left.1+\gamma_{k}\right) d_{i j}^{\alpha_{0}}+e_{i j}$,

with $S p p$ included as a fixed factor in the coefficient, represented by $\gamma_{k}, k=$ $1,2,3$.

- euc.hd.8: $h_{i j}=\left(\beta_{0}+b_{0 i}+\beta_{1}\right.$ Age $1+\beta_{2}$ Stock 2$) d_{i j}^{\alpha_{0}}+e_{i j}$, now we have included Stock 2 as a fixed effect in the coefficient, with its own coefficient $\beta_{2}$.

- euc.hd.9: $h_{i j}=\left(\beta_{0}+\left(\beta_{1}+b_{1 i}\right)\right.$ Age $1+\beta_{2}$ Stock $\left.2+\gamma_{k}\right) d_{i j}^{\alpha_{0}}+e_{i j}$, using both stocking and species in the model.

- euc.hd.10:

$h_{i j}=\left(\beta_{0}+\left(\beta_{1}+b_{1 i}\right)\right.$ Age $1+\beta_{2}$ Stock 2$) d_{i j}^{\alpha_{0}}+e_{i j}$,

185 now we include a random coefficient of Age 1 in the coefficient of $d$. We assume (4) that the $b_{1 i} \sim$ i.i.d. $\mathcal{N}\left(0, \sigma_{b_{1}}^{2}\right)$, and are independent of the $e_{i j} s$.

- euc.hd.11:

$h_{i j}=\left(\beta_{0}+b_{0 i}+\left(\beta_{1}+b_{1 i}\right)\right.$ Age $1+\beta_{2}$ Stock 2$) d_{i j}^{\alpha_{0}}+e_{i j}$,

where we assume $(6)$ that the $b_{0 i} \sim$ i.i.d. $\mathcal{N}\left(0, \sigma_{b_{0}}^{2}\right)$ and are independent of the $190 \quad e_{i j} \mathrm{~s}$.

- euc.hd.12:

$h_{i j}=\left(\beta_{0}+b_{1 i}\right.$ Age $1+\left(\beta_{2}+b_{2 i}\right)$ Stock 2$) d_{i j}^{\alpha_{0}}+e_{i j}$,

- euc.hd.13:

$h_{i j}=\left(\beta_{0}+\beta_{1}\right.$ Age $1+\beta_{3}$ Stock 2$\left.) d_{i j}^{(} \alpha_{0}+a_{0 i}\right)+e_{i j}$,

195

- euc.hd.14:

$h_{i j}=\left(\beta_{0}+b_{0 i} \beta_{1} A g e 1+\beta_{2} A g e 1^{2}\right) d_{i j}^{\alpha_{0}}+e_{i j}$,

- euc.hd.15: as euc.hd.14, with a separate variance estiamted for each site's residuals.

- euc.hd.16:

$h_{i j}=\left(\beta_{0}+b_{0 i}+\beta_{1} A g e 1^{2}\right) d_{i j}^{\alpha_{0}}+e_{i j}$,

with a separate variance estimated for each site's residuals. 
- euc.hd.17:

$h_{i j}=\left(\beta_{0}+b_{0 i}+\left(\beta_{1}+b_{2 i}\right) A g e 1^{2}\right) d_{i j}^{\alpha_{0}}+e_{i j}$,

with a separate variance estimated for each site's residuals.

205

- euc.hd.18:

$h_{i j}=\left(\beta_{0}+b_{0 i}+\beta_{1}\right.$ Age $1+\left(\beta_{2}+b_{2 i}\right) A g e 1^{2}+\beta_{3}$ Stock 2$) d_{i j}^{\alpha_{0}}+e_{i j}$,

with a separate variance estimated for each site's residuals.

- euc.hd.19:

$h_{i j}=\left(\beta_{0}+b_{0 i}+\beta_{1}\right.$ Age $1+\left(\beta_{2}+b_{2 i}\right) A g e 1^{2}+\beta_{3}$ Stock $\left.2+\gamma_{k}\right) d_{i j}^{\alpha_{0}}+e_{i j}$, with a separate variance estimated for each site's residuals.

\subsubsection{Height vs. diameter}

Using nonlinear least squares, the model that best described the relationship between height and diameter for the Eucal dataset described the height of the $j^{\text {th }}$ tree of species $k$ in the $i^{t h}$ site as

$$
h t_{i j}=\left(\beta_{0}+\left(\beta_{1}+b_{1 i}\right) \sqrt{a g e 1}+\beta_{2} \text { stocking } 2+x_{k}\right) d o b 1.3^{\left(\alpha_{0}+\alpha_{1} \sqrt{a g e 1}\right)}+\epsilon_{i j}
$$

with $\operatorname{Var}\left(\epsilon_{i j}\right)=\sigma^{2}\left|a g e 1_{i j}\right|^{2 \delta}$, in which the exponent of diameter varies with the age 1 covariate.

Table 2.2: Parameter estimates and confidence intervals for model Eucal.hd

\begin{tabular}{|c|c|c|}
\hline Parameter & Estimate & Approx. 95\% C.I. \\
\hline$\beta_{0}$ & -0.377 & $(-0.650,-0.104)$ \\
$\beta_{1}$ & 6.268 & $(5.400,7.136)$ \\
$\sigma_{b_{1}}$ & 0.868 & $(0.610,1.235)$ \\
$\beta_{2}$ & -0.250 & $(-0.356,-0.143)$ \\
$x$ & 0 for E. globulus & - \\
& -0.261 for E. grandis & $(-0.337,-0.186)$ \\
$\alpha_{0}$ & 0.597 & $(0.562,0.633)$ \\
$\alpha_{1}$ & -0.187 & $(-0.218,-0.155)$ \\
$\delta$ & 0.631 & $(0.531,0.731)$ \\
$\sigma$ & 1.759 & $(1.571,1.969)$ \\
\hline
\end{tabular}

The within-group errors are estimated as $\hat{\operatorname{Var}}\left(\epsilon_{i j}\right)=1.759^{2} \mid$ age $\left.1_{i j}\right|^{2 \times 0.631}$. The 215 variance within each group increases as age 1 does with a power of 1.262. 


\subsubsection{Mass vs. diameter}

Using nonlinear least squares, the model that best described the relationship between mass and diameter for the Eucal dataset described the mass of the $j^{\text {th }}$ tree of species $k$ in the $i^{\text {th }}$ site as

$$
m_{i j}=\left(\beta_{0}+b_{0 i}+\beta_{1} \text { age } 1+\beta_{2} a g e 1^{2}+\beta_{3} \text { stocking } 2\right) d o b 1.3^{\left(\alpha_{0}+\alpha_{1} a g e 1^{2}+x_{k}\right)}+\epsilon_{i j}
$$

with $\operatorname{Var}\left(\epsilon_{i j}\right)=\sigma^{2}\left|a g e 1_{i j}\right|^{2 \delta}$, and in which the exponent of diameter varies with age 1 and species.

Table 2.3: Parameter estimates and confidence intervals for model Eucal.md

\begin{tabular}{|c|c|c|}
\hline Parameter & Estimate & Approx. 95\% C.I. \\
\hline$\beta_{0}$ & 0.169 & $(0.132,0.206)$ \\
$\sigma_{b_{0}}$ & 0.0285 & $(0.0174,0.0465)$ \\
$\beta_{1}$ & 0.229 & $(0.097,0.361)$ \\
$\beta_{2}$ & -0.296 & $(-0.396,-0.196)$ \\
$\beta_{3}$ & -0.0049 & $(-0.0071,-0.0027)$ \\
$\alpha_{0}$ & 2.108 & $(2.029,2.186)$ \\
$\alpha_{1}$ & 0.399 & $(0.279,0.518)$ \\
$x$ & 0 for E. globulus & - \\
& -0.036 for E. grandis & $(-0.051,-0.021)$ \\
$\delta$ & 1.328 & $(1.235,1.421)$ \\
$\sigma$ & 24.54 & $(22.13,27.22)$ \\
\hline
\end{tabular}

The within-group errors are estimated as $\hat{\operatorname{Var}}\left(\epsilon_{i j}\right)=24.54^{2} \mid$ age $\left.1_{i j}\right|^{2 \times 1.328}$. The variance within each group increases as age1 does with a power of 2.656.

\subsubsection{Height vs. mass}

Using nonlinear least squares, the best-fitting model to describe the relationship between the height and mass of trees in the Eucal dataset described the height of the $j^{\text {th }}$ tree in the $i^{\text {th }}$ site as

$$
h t_{i j}=\left(\beta_{0}+\left(\beta_{1}+b_{1 i}\right) \sqrt{a g e 1}+\beta_{2} \text { stocking } 2+\beta_{3} M i n T\right) m^{\left(\alpha_{0}+\alpha_{1} \sqrt{a g e 1}\right)}+\epsilon_{i j}
$$

with $\operatorname{Var}\left(\epsilon_{i j}\right)=\sigma^{2}\left|a g e 1_{i j}\right|^{2 \delta}$, MinT represents the minimum average monthly temperature in degrees Celsius, and in which the exponent of mass varies with age1.

The within-group errors are estimated as $\hat{\operatorname{Var}}\left(\epsilon_{i j}\right)=1.660^{2} \mid$ age $\left.1_{i j}\right|^{2 \times 0.590}$. The variance within each group increases as age 1 does with a power of 1.180. 
Table 2.4: Parameter estimates and confidence intervals for model Eucal.hm

\begin{tabular}{|c|c|c|}
\hline Parameter & Estimate & Approx. 95\% C.I. \\
\hline$\beta_{0}$ & -1.291 & $(-1.793,-0.789)$ \\
$\beta_{1}$ & 8.830 & $(7.901,9.760)$ \\
$\sigma_{b_{1}}$ & 1.044 & $(0.627,1.737)$ \\
$\beta_{2}$ & -0.370 & $(-0.509,-0.232)$ \\
$\beta_{3}$ & 0.146 & $(0.018,0.273)$ \\
$\alpha_{0}$ & 0.337 & $(0.318,0.355)$ \\
$\alpha_{1}$ & -0.161 & $(-0.179,-0.144)$ \\
$\delta$ & 0.590 & $(0.484,0.697)$ \\
$\sigma$ & 1.660 & $(1.475,1.867)$ \\
\hline
\end{tabular}




\section{Diagnostic plots for fitted models}

\section{1 abies.2.4 - height vs. diameter}

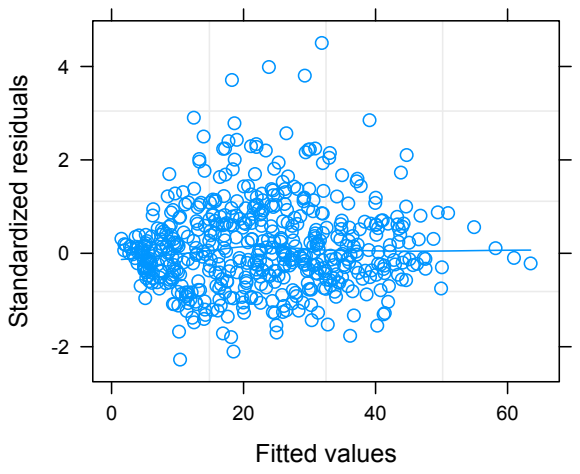

(a) Standardized residuals vs. fitted values

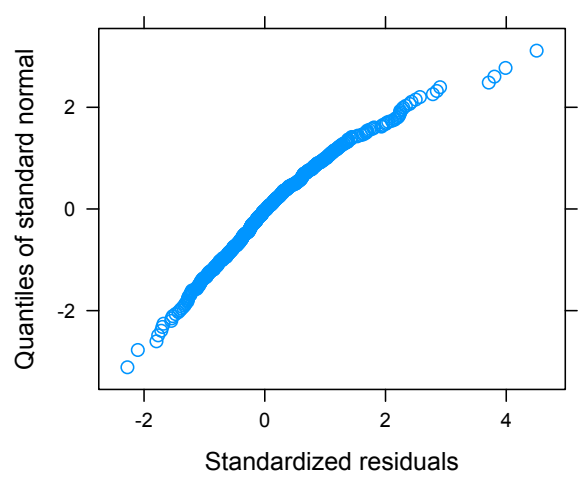

(c) QQ-plot of standardized residuals

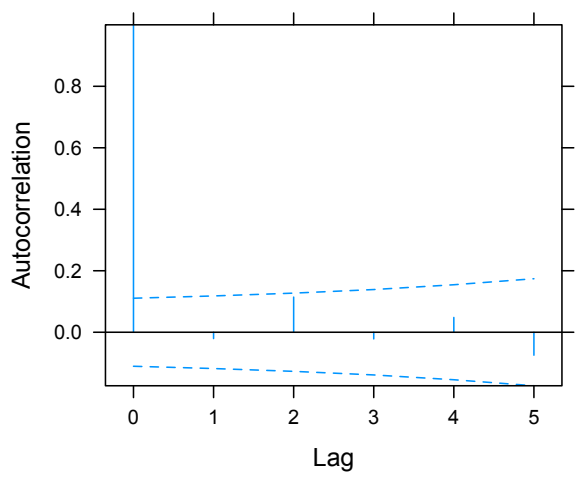

(b) Autocorrelation function

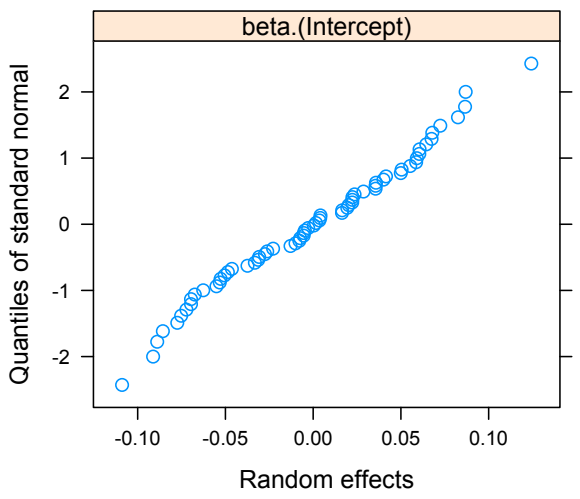

(d) QQ-plot of random effects

Figure 3.1: Diagnostic plots for model abies.2.4 - (a) These residuals are not ideal in that there appears to be some kind of pattern still present in the residuals. However, these were the best-looking residuals produced by any model fit. (b) The autocorrelation function shows the correlation between adjacent observations to be small. (c) The residuals appear to be evenly scattered around a straight line, displaying enough correspondence with a sample of ordered points drawn from a normal distribution to satisfy the assumption of normal residuals. (d) The random effects are closely scattered around a straight line, satisfying the assumption of normal random effects. 

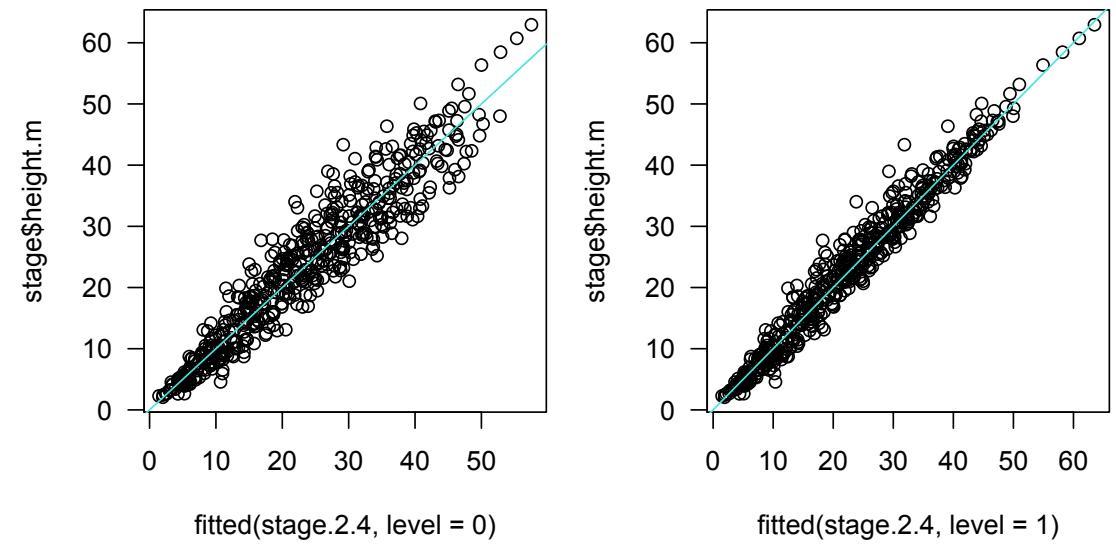

Figure 3.2: Observations vs. fitted values without and with random effects - The inclusion of the random effect in the model (level $=1$ ) enables a closer correspondence between the observed and fitted heights than without (level $=0$ ).

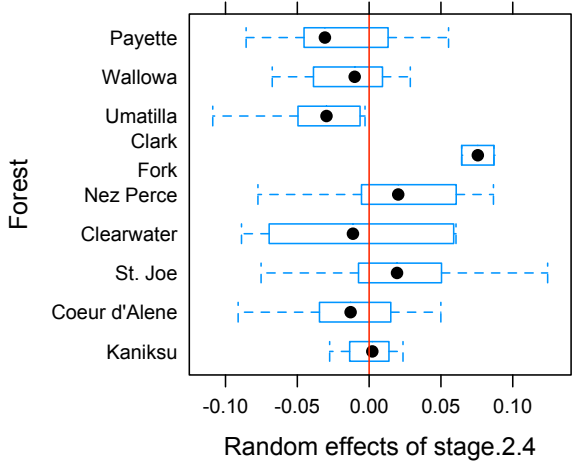

(a) Random effects for each forest

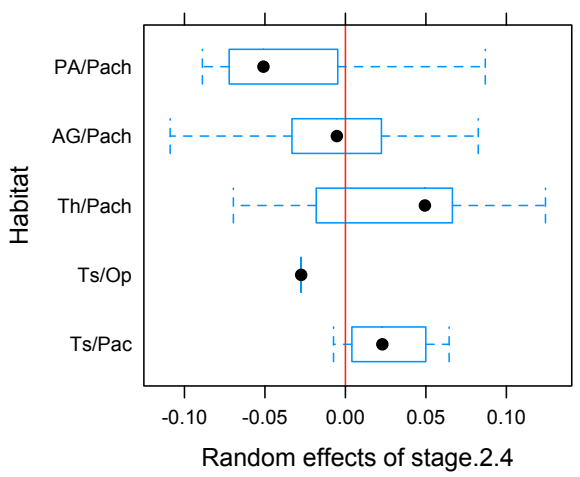

(b) Random effects for each habitat

Figure 3.3: Boxplots of the random effects of model abies.2.4 for each forest and habitat - The boxplots for the forest Clark Fork and habitat Ts/Op come from two and one trees respectively, so their distance from zero (the red line) can be disregarded. The other random effects boxplots appear to overlap enough to conclude that further random effects for forest and habitat need not be added to the model. 


\subsection{Eucal.hd - height vs. diameter}

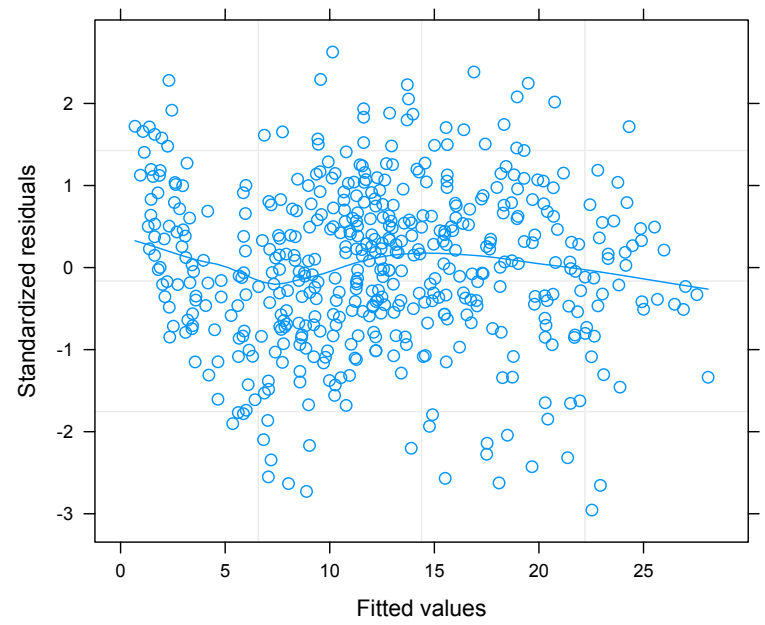

Figure 3.4: Standardized residuals vs. fitted values - The standardized residuals are evenly scattered around zero and patternless, satisfying the assumption of independent and identically distributed residuals.

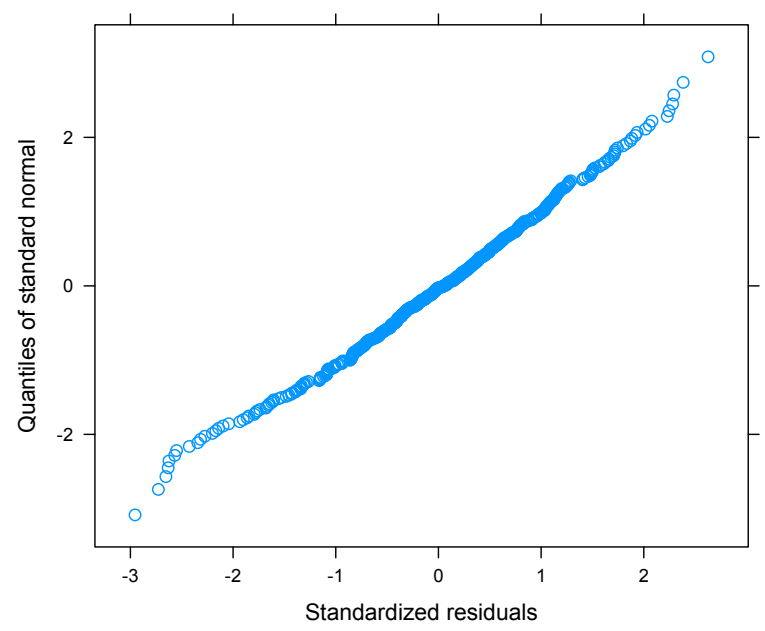

Figure 3.5: QQ-plot of standardized residuals — The standardized residuals are evenly scattered around a straight line, satisfying the assumption of normal residuals. 


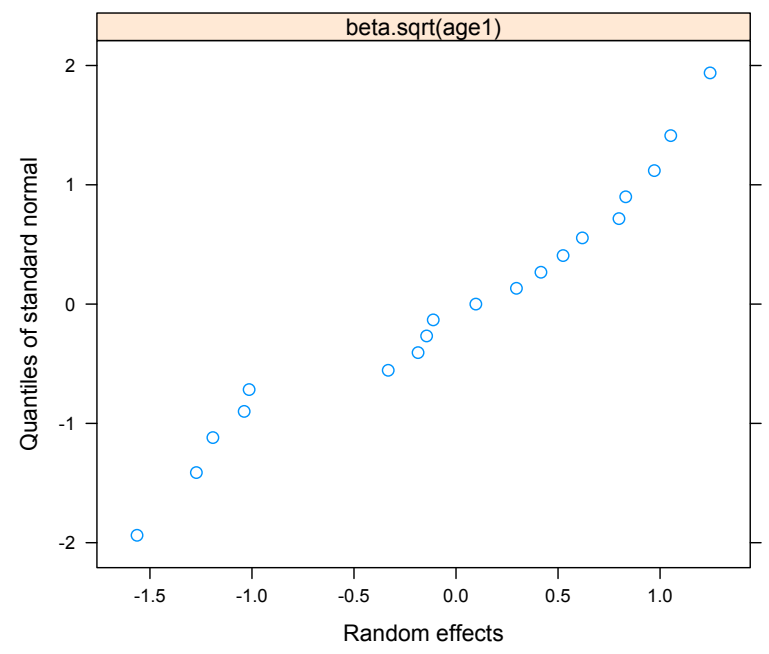

Figure 3.6: QQ-plot of random effects - The random effects are evenly scattered around a straight line, satisfying our assumption of their normality. 

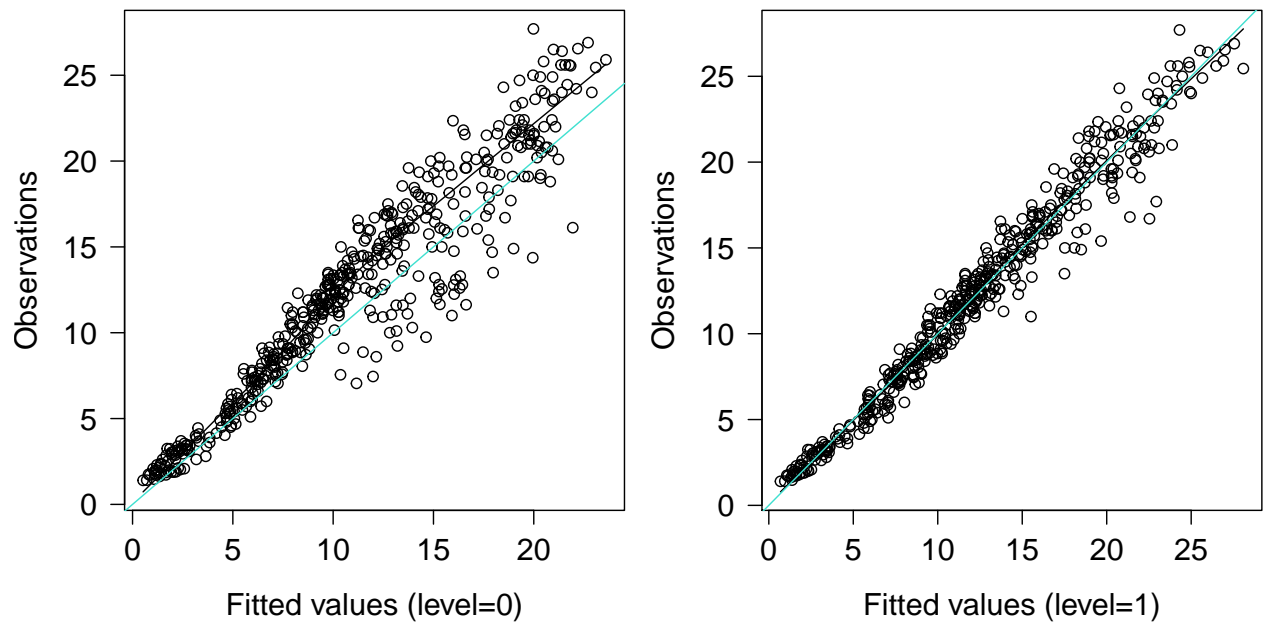

Figure 3.7: Observations vs. fitted values without and with random effects - The random effects (level $=1$ ) enable a closer correspondence between the observations and fitted values than without (level $=0$ ).

\subsection{Eucal.md - mass vs. diameter}




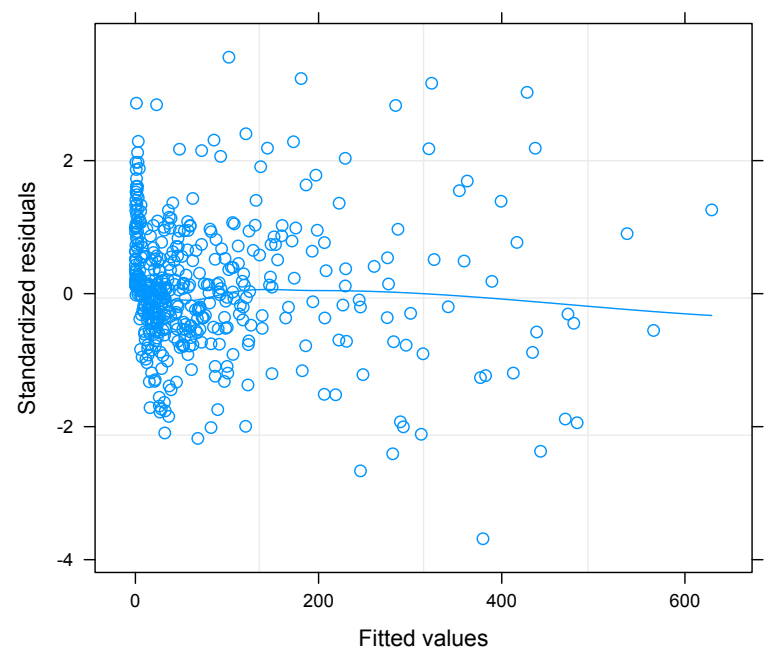

Figure 3.8: Standardized residuals vs. fitted values - The standardized residuals are evenly scattered around zero and patternless, satisfying the assumption of independent and identically distributed residuals.

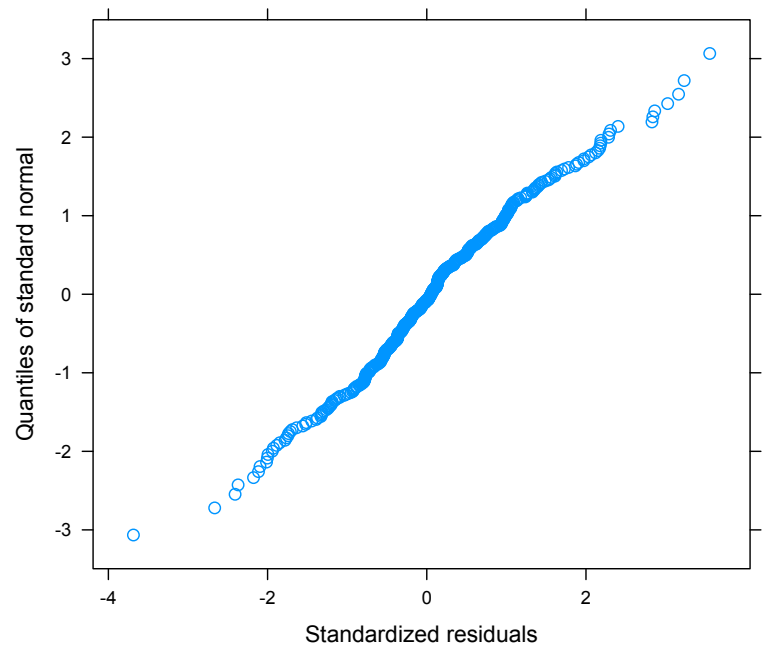

Figure 3.9: QQ-plot of standardized residuals — The standardized residuals are evenly scattered around a straight line, satisfying the assumption of normal residuals.

230 3.4 Eucal.hm — height vs. mass 


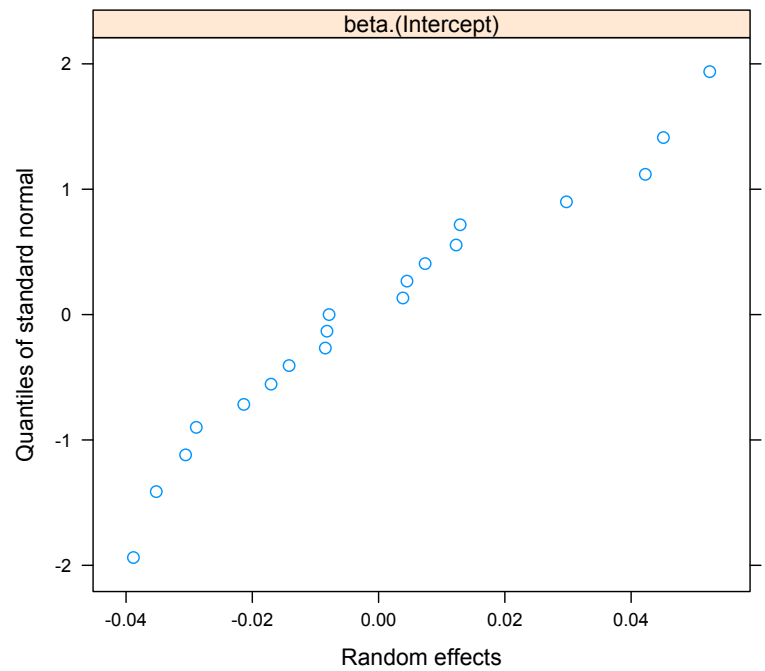

Figure 3.10: QQ-plot of random effects - The random effects are evenly scattered around a straight line, satisfying our assumption of their normality. 

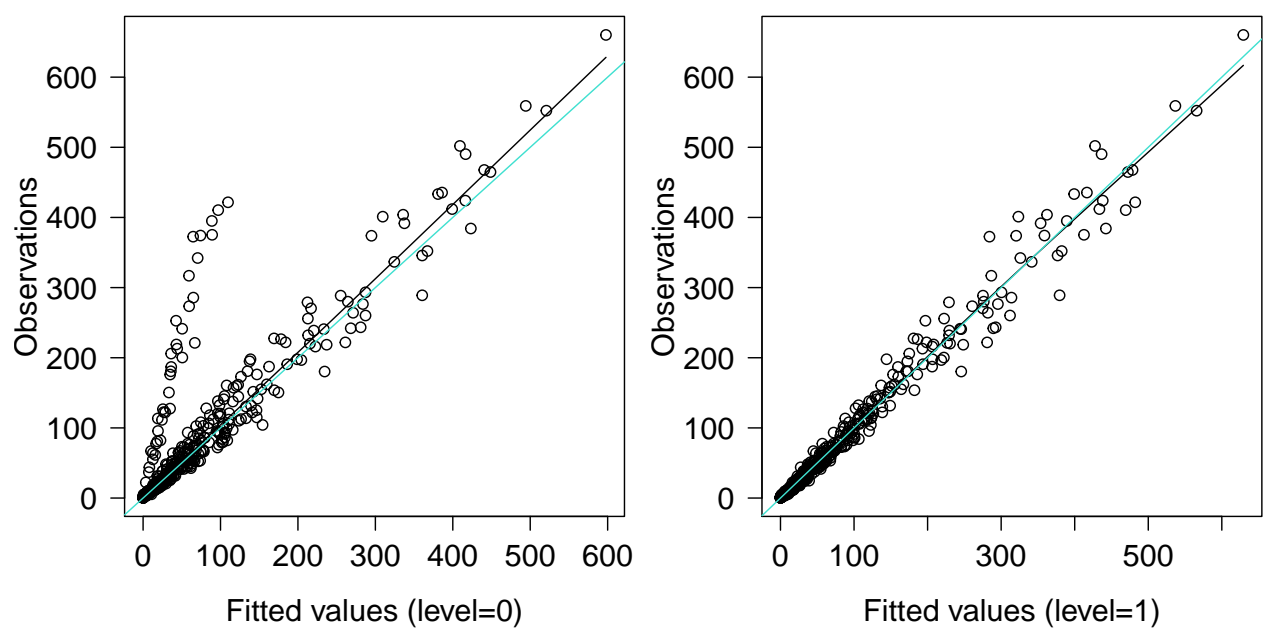

Figure 3.11: Observations vs. fitted values without and with random effects - The random effects (level $=1$ ) enable a closer correspondence between the observations and fitted values than without (level $=0$ ), particularly the string of fitted values less than $100 \mathrm{~kg}$. 


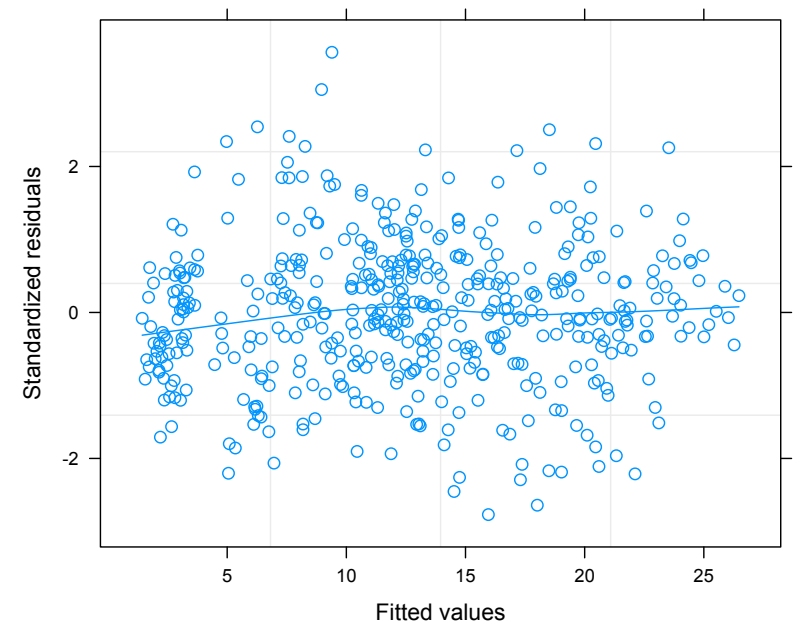

Figure 3.12: Standardized residuals vs. fitted values — The standardized residuals are evenly scattered around zero and patternless, satisfying the assumption of independent and identically distributed residuals.

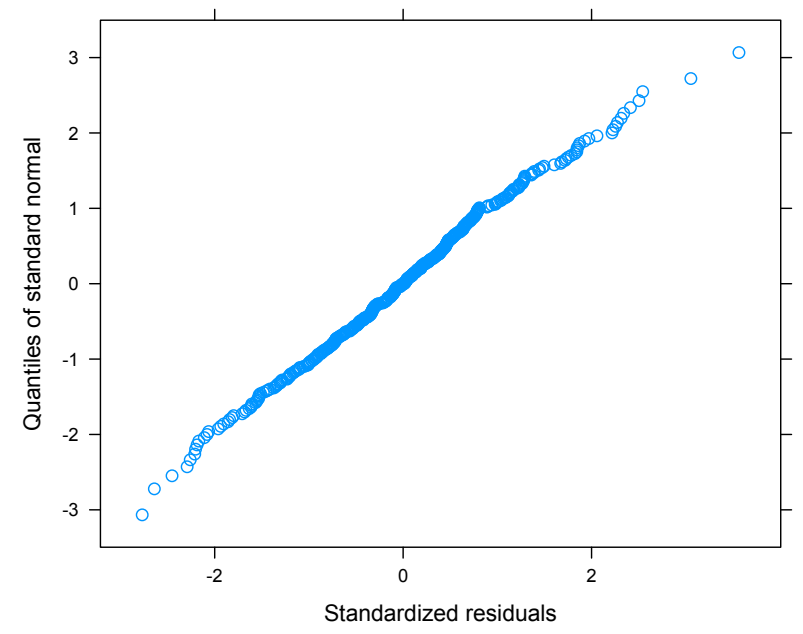

Figure 3.13: QQ-plot of standardized residuals - The standardized residuals are evenly scattered around a straight line, satisfying the assumption of normal residuals. 


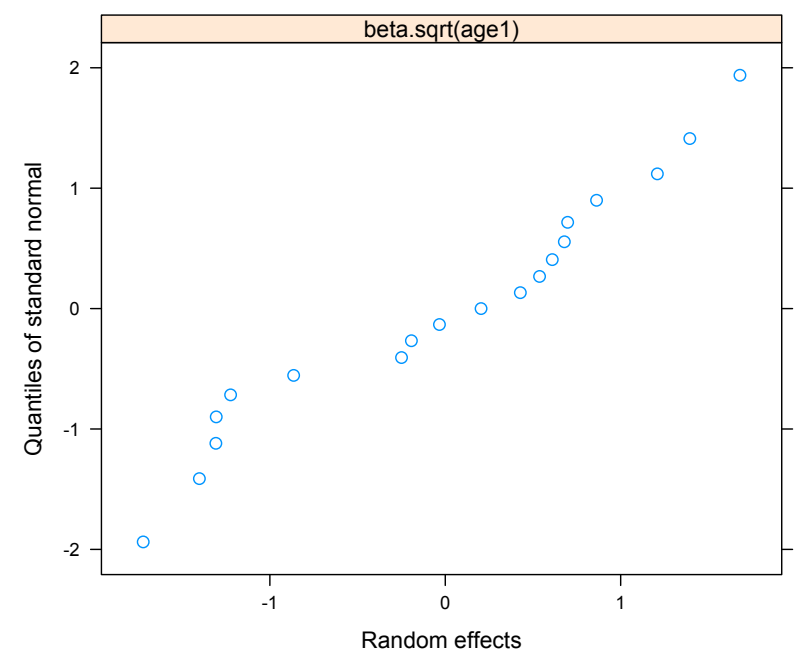

Figure 3.14: QQ-plot of random effects - The random effects are evenly scattered around a straight line, satisfying our assumption of their normality. 

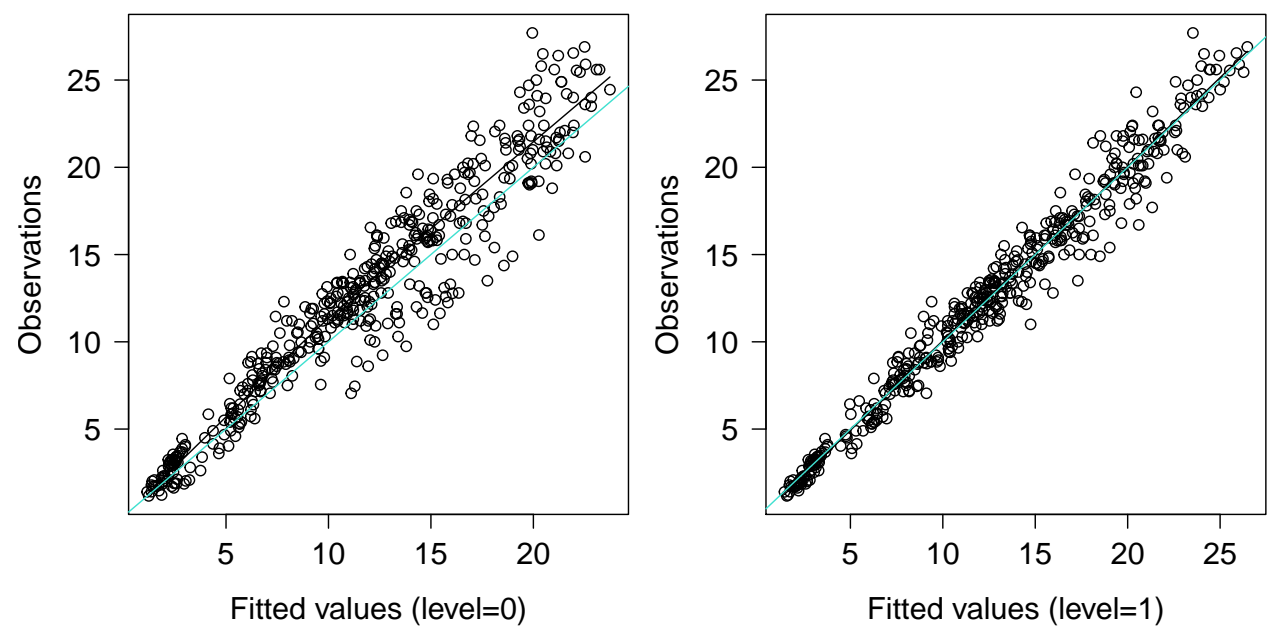

Figure 3.15: Observations vs. fitted values without and with random effects - The random effects (level $=1$ ) enable a closer correspondence between the observations and fitted values than without (level $=0$ ). 


\section{Details of the regression-based equivalence test}

1. Observe $y_{o i}$ from the data, and predict $y_{p i}$ from the proposed model, $i=$ $1, \ldots, n$.

2. Calculate $\bar{y}_{o}=\frac{1}{n} \sum_{i=1}^{n} y_{o i}$ and $\bar{y}_{p}=\frac{1}{n} \sum_{i=1}^{n} y_{p i}$.

3. Calculate $x=y_{p}-\bar{y}_{p}$. With this shift:

- The slope when $x$ is regressed against $y_{o}$ will be the same as if $y_{p}$ is regressed against $y_{0}$.

- Estimates of intercept $\left(\beta_{0}\right)$ and slope $\left(\beta_{1}\right)$ will be independent by construction when $x$ is regressed against $y_{o}$.

- If the predictions $y_{p}$ are unbiased then $\beta_{0}=\bar{y}_{o}$.

4. Establish regions of equivalence, or indifference, $I$.

- For the shifted intercept $\beta_{0}, I_{0}=\bar{y}_{p} \pm \delta_{0}$.

- For the slope $\beta_{1}, I_{1}=1 \pm \delta_{1}$.

- The regions of equivalence allow for the incorporation of practical significance into the tests. Both $\delta_{0}$ and $\delta_{1}$ can be selected on the basis of a difference between prediction and observation that is irrelevant, or ignorable, in the real world context of the problem. They can be absolute values, or proportions of $\bar{y}_{p}$.

5. Decide on the desired joint size of the test of the model, $\alpha_{j}$.

- Since two parameters are being tested within one model, a correction must be made with regard to size.

- For example, if the desired size for each individual test is $\alpha_{i}=0.05$, then the joint size for the two independent tests is

$$
\begin{aligned}
\alpha_{j} & =1-\left(1-\alpha_{i}\right)^{2} \\
& =1-(1-0.05)^{2} \\
& =0.0975 \\
& \geqslant \alpha_{i} .
\end{aligned}
$$

To achieve a joint size of $\alpha_{j}=0.05$, rearrangement gives the individual 
test size

$$
\begin{aligned}
\alpha_{i} & =1-\left(1-\alpha_{j}\right)^{\frac{1}{2}} \\
& =1-(1-0.05)^{\frac{1}{2}} \\
& =0.02532 \\
& \leqslant \alpha_{j} .
\end{aligned}
$$

6. Regress $y_{o}$ (as the response) against $x$ (as the predictor).

7. Test $H_{0}: \beta_{0} \neq \bar{y}_{o}$ against $H_{1}: \beta_{0}=\bar{y}_{o}$.

- This tests whether the mean of the observations is equivalent to the mean of the predictions. In other words, this tests for a lack of bias.

- To test, construct two one-sided $\left(1-\alpha_{i}\right) \%$ confidence intervals for $\beta_{0}$ at $x=0$, where $\alpha_{i}$ is obtained as described above. The estimated standard error of the intercept from the regression summary can be used for this.

- If, at $x=0$, these confidence intervals are contained completely within $I_{0}$, reject $H_{0}$. If not, there is not sufficient evidence to conclude that $\beta_{0}=\bar{y}_{o}$ (i.e. that the predictions and model are unbiased).

8. Test $H_{0}: \beta_{1} \neq 1$ against $H_{1}: \beta_{1}=1$.

- This tests whether the predictions are sufficiently in accord with the observations. In other words, this tests the accuracy of the model.

- To test, construct two one-sided $\left(1-\alpha_{i}\right) \%$ confidence intervals for $\beta_{1}$, where $\alpha_{i}$ is as previously described. The estimated standard error of the slope from the regression summary can be used for this.

- If these confidence intervals are contained completely within $I_{1}$, reject $H_{0}$. If not, there is not sufficient evidence to conclude that the predictions are sufficiently similar to the observations.

9. Obtaining the confidence intervals.

- It may not always be possible to use parametric methods to generate confidence intervals for these parameters. For example, key regression assumptions may be violated, such as the correctness of the specified model or the independence or distribution of the errors. If any of these problems occur, asymptotically obtained confidence intervals become unreliable. To avoid such difficulties, non-parametric bootstrapping can be used to obtain the needed confidence intervals as described below. 
(a) To create $b$ bootstrap replicates, select $n$ observation pairs (an observation $y_{o i}$ and its corresponding prediction $y_{p i}$ ) with replacement from the original sample.

(b) For each of the $b$ bootstrap replicates, regress the observations against predictions as described earlier, and thus obtain $b$ estimates of $\beta_{0}$ and $\beta_{1}$.

(c) Calculate the proportion of times that $\hat{\beta}_{0}$ is within $I_{0}$, and the proportion of times that $\hat{\beta}_{1}$ is within $I_{1}$.

(d) Record the empirical quantiles corresponding to the joint two onesided $\left(1-\alpha_{i}\right) \%$ confidence intervals for slope and intercept for the desired size $\alpha_{j}$.

(e) If for either the slope or estimate the proportion of times that the estimate was within the region of equivalence is greater than $1-(2 \times$ $\left.\alpha_{i}\right)$, reject the null hypothesis that the predictions and the data are dissimilar.

The equivalence package in $R$ performs these tests. 


\section{Calculations of standard errors and confidence intervals for the exponents of models from the Eucal dataset}

\subsection{Height vs. diameter}

In each of these instances, the standard errors of the parameter estimates were obtained from the summary output of $\mathrm{R}$. The covariances were obtained using the vcov command, and the degrees of freedom used were also taken from the summary output. The degrees of freedom are only approximate, but of large enough size that the quantile values should not vary much regardless. The standard error of the estimated exponent was

$$
\begin{aligned}
\text { s.e. }\left(\hat{\alpha_{0}}+\hat{\alpha_{1}} \sqrt{a g e 1}\right) & =\sqrt{\operatorname{vâr}\left(\hat{\alpha_{0}}+\hat{\alpha_{1}} \sqrt{a g e 1}\right)} \\
& =\sqrt{\operatorname{vâr}\left(\hat{\alpha_{0}}\right)+\operatorname{age} 1 \operatorname{vâr}\left(\hat{\alpha_{1}}\right)+2 \sqrt{a g e 1} \operatorname{cov} \operatorname{var}\left(\hat{\alpha_{0}}, \hat{\alpha_{1}}\right)} \\
& =\sqrt{\text { s.e. }\left(\hat{\alpha_{0}}\right)^{2}+\operatorname{age} 1 \text { s.e. }\left(\hat{\alpha_{1}}\right)^{2}+2 \sqrt{\operatorname{age} 1} \operatorname{cov} \operatorname{ar}\left(\hat{\alpha_{0}}, \hat{\alpha_{1}}\right)} \\
& =\sqrt{0.000335+0.000263 a g e 1-0.000305 \sqrt{a g e 1}} .
\end{aligned}
$$

The two one-sided $95 \%$ confidence interval for the exponent was calculated as

$$
\begin{aligned}
& \hat{\alpha_{0}}+\hat{\alpha_{1}} \sqrt{\text { age } 1} \pm t_{0.95,466} \times \text { s.e. }\left(\hat{\alpha_{0}}+\hat{\alpha_{1}} \sqrt{\text { age } 1}\right) \\
& =0.597-0.187 \sqrt{\text { age } 1} \pm 1.648 \times \sqrt{0.000335+0.000263 a g e 1-0.000305 \sqrt{\text { age } 1}} \\
& =\left\{\begin{array}{l}
(0.511,0.565) \text { for the minimum age in decades, } 0.1, \\
(0.363,0.422) \text { for the maximum age in decades, } 1.2 .
\end{array}\right.
\end{aligned}
$$

\subsection{Mass vs. diameter}

For E. globulus the variance of the estimated exponent $\hat{\alpha_{0}}+\hat{\alpha_{1}} a g e 1^{2}+s \hat{p} p$ was

$$
\begin{aligned}
& \operatorname{vâr}\left(\hat{\alpha_{0}}+\hat{\alpha_{1}} a g e 1^{2}+s \hat{p} p\right)=\operatorname{vâr}\left(\hat{\alpha_{0}}\right)+a g e 1^{4} \operatorname{vâr}\left(\hat{\alpha_{1}}\right)+\operatorname{vâr}(E \cdot g l \hat{o b u l u s}) \\
& +2 a g e 1^{2} \operatorname{cov} \operatorname{var}\left(\hat{\alpha_{0}}, \hat{\alpha_{1}}\right)+2 \operatorname{cov} a r\left(\hat{\alpha_{0}}, \text { E. globulus }\right) \\
& +2 a g e 1^{2} \operatorname{cov} a r\left(\hat{\alpha_{1}}, \text { E. glôbulus }\right) \\
& =0.00164-0.00215 \text { age } 1^{2}+0.00375 a g e 1^{4} \\
& \Rightarrow \text { s.e. }\left(\hat{\alpha_{0}}+\hat{\alpha_{1}} a g e 1^{2}+s \hat{p} p\right)=\sqrt{0.00164-0.00215 a g e 1^{2}+0.00375 a g e 1^{4}} \text {. }
\end{aligned}
$$


The two one-sided 95\% confidence interval for the exponent was calculated as

$$
\begin{aligned}
& \hat{\alpha_{0}}+\hat{\alpha_{1}} a g e 1^{2}+s \hat{p} p \pm t_{0.95,435} \times \text { s.e. }\left(\hat{\alpha_{0}}+\hat{\alpha_{1}} a g e 1^{2}+s \hat{p} p\right) \\
& =2.108+0.399 a g e 1^{2} \pm 1.648 \times \sqrt{0.00164-0.00215 a g e 1^{2}+0.00375 a g e 1^{4}} \\
& =\left\{\begin{array}{l}
(2.045,2.178) \text { for the minimum age in decades, } 0.1, \\
(2.551,2.813) \text { for the maximum age in decades, } 1.2 .
\end{array}\right.
\end{aligned}
$$

For E. grandis the variance of the estimated exponent was

$$
\begin{aligned}
& \operatorname{vâr}\left(\hat{\alpha_{0}}+\hat{\alpha_{1}} a g e 1^{2}+s \hat{p} p\right)=\operatorname{vâr}\left(\hat{\alpha_{0}}\right)+a g e 1^{4} \operatorname{vâr}\left(\hat{\alpha_{1}}\right)+\operatorname{vâr}(\text { E. grandis }) \\
&+2 a g e 1^{2} \operatorname{cov} \hat{\operatorname{var}}\left(\hat{\alpha_{0}}, \hat{\alpha_{1}}\right)+2 \operatorname{cov} \hat{\operatorname{var}}\left(\hat{\alpha_{0}}, \text { E. grandis }\right) \\
&+2 a g e 1^{2} \operatorname{cov} \hat{\operatorname{var}}\left(\hat{\alpha_{1}}, E \cdot \text { grandis }\right) \\
&= 0.00164-0.00215 a g e 1^{2}+0.00375 a g e 1^{4} \\
& \Rightarrow \text { s.e. }\left(\hat{\alpha_{0}}+\hat{\alpha_{1}} a g e 1^{2}+s \hat{p} p\right)=\sqrt{0.00185-0.00214 a g e 1^{2}+0.00375 a g e 1^{4}} .
\end{aligned}
$$

The two one-sided $95 \%$ confidence interval for the exponent was calculated as

$$
\begin{aligned}
& \hat{\alpha_{0}}+\hat{\alpha_{1}} \text { age } 1^{2}+s \hat{p} p \pm t_{0.95,435} \times \text { s.e. }\left(\hat{\alpha_{0}}+\hat{\alpha_{1}} a g e 1^{2}+s \hat{p} p\right) \\
& =2.108+0.399 a g e 1^{2}-0.0360 \pm 1.648 \times \sqrt{0.00185-0.00214 a g e 1^{2}+0.00375 a g e 1^{4}} \\
& =\left\{\begin{array}{l}
(2.005,2.146) \text { for the minimum age in decades, } 0.1, \\
(2.512,2.779) \text { for the maximum age in decades, } 1.2 .
\end{array}\right.
\end{aligned}
$$

\section{$300 \quad 5.3 \quad$ Height vs. mass}

The standard error of the estimated exponent $\hat{\alpha_{0}}+\hat{\alpha_{1}} \sqrt{a g e 1}$ was

$$
\begin{aligned}
\text { s.e. }\left(\hat{\alpha_{0}}+\hat{\alpha_{1}} \sqrt{a g e 1}\right) & =\sqrt{\operatorname{vâr}\left(\hat{\alpha_{0}}+\hat{\alpha_{1}} \sqrt{a g e 1}\right)} \\
& =\sqrt{\operatorname{vâr}\left(\hat{\alpha_{0}}\right)+\operatorname{age} 1 \operatorname{vâr}\left(\hat{\alpha_{1}}\right)+2 \sqrt{a g e 1} \operatorname{cov} \operatorname{ar}\left(\hat{\alpha_{0}}, \hat{\alpha_{1}}\right)} \\
& =\sqrt{\text { s.e. }\left(\hat{\alpha_{0}}\right)^{2}+\operatorname{age} 1 \text { s.e. }\left(\hat{\alpha_{1}}\right)^{2}+2 \sqrt{\operatorname{age} 1} \operatorname{cov} \hat{\operatorname{var}}\left(\hat{\alpha_{0}}, \hat{\alpha_{1}}\right)} \\
& =\sqrt{0.0000907+0.0000766 a g e 1-0.000118 \sqrt{a g e 1}} .
\end{aligned}
$$


The two one-sided 95\% confidence interval for the exponent was calculated as

$$
\begin{aligned}
& \hat{\alpha_{0}}+\hat{\alpha_{1}} \sqrt{\text { age } 1} \pm t_{0.95,438} \times \text { s.e. }\left(\hat{\alpha_{0}}+\hat{\alpha_{1}} \sqrt{\text { age } 1}\right) \\
& =0.337-0.161 \sqrt{\text { age } 1} \pm 1.648 \times \sqrt{0.0000907+0.0000766 a g e 1-0.000118 \sqrt{\text { age } 1}} \\
& =\left\{\begin{array}{l}
(0.273,0.299) \text { for the minimum age in decades, } 0.1, \\
(0.148,0.172) \text { for the maximum age in decades, } 1.2 .
\end{array}\right.
\end{aligned}
$$

\subsection{Leaf area vs. diameter}

The variance of the estimated exponent $\hat{\alpha_{0}}+\hat{a_{0 i}}+\hat{\alpha_{1}}$ age $1+\hat{\alpha_{2}}$ stocking 2 was calculated as follows. To make the calculations easier, we replaced age 1 with $x$ and stocking 2 with $y$.

$$
\begin{aligned}
\operatorname{vâr}\left(\hat{\alpha_{0}}+\hat{a_{0 i}}+\hat{\alpha_{1}} x+\hat{\alpha_{2} y}\right)=\operatorname{vâr}\left(\hat{\alpha_{0}}\right)+\operatorname{vâr}\left(\hat{a_{0 i}}\right)+x^{2} \operatorname{vâr}\left(\hat{\alpha_{1}}\right)+y^{2} \operatorname{vâr}\left(\hat{\alpha_{2}}\right) \\
+\operatorname{cov} \hat{\operatorname{var}}\left(\hat{\alpha_{0}}, \hat{a_{0 i}}\right)+2 x \operatorname{cov} \operatorname{var}\left(\hat{\alpha_{0}}, \hat{\alpha_{1}}\right) \\
+2 y \operatorname{cov} \hat{\operatorname{var}}\left(\hat{\alpha_{0}}, \hat{\alpha_{2}}\right)+2 x \operatorname{cov} \operatorname{var}\left(\hat{a_{0 i}}, \hat{\alpha_{1}}\right) \\
+2 y \operatorname{covar}\left(\hat{a_{0 i}}, \hat{\alpha_{2}}\right)+2 x y \operatorname{cov} \operatorname{var}\left(\hat{\alpha_{1}}, \hat{\alpha_{2}}\right) \\
=\operatorname{vâr}\left(\hat{\alpha_{0}}\right)+\hat{\sigma_{a 0}^{2}}+x^{2} \operatorname{vâr}\left(\hat{\alpha_{1}}\right)+y^{2} \operatorname{vâr}\left(\hat{\alpha_{2}}\right) \\
+2 x \operatorname{cov} \operatorname{var}\left(\hat{\alpha_{0}}, \hat{\alpha_{1}}\right)+2 y \operatorname{covar}\left(\hat{\alpha_{0}}, \hat{\alpha_{2}}\right) \\
+2 x y \operatorname{cov} \hat{\operatorname{var}}\left(\hat{\alpha_{1}}, \hat{\alpha_{2}}\right)
\end{aligned}
$$

(since the fixed and random effects are independent and have zero covariance)

$$
\begin{gathered}
=\left\{\begin{array}{c}
\operatorname{vâr}\left(\hat{\alpha_{0}}\right)+\hat{\sigma_{a 0}^{2}}+x^{2} \operatorname{vâr}\left(\hat{\alpha_{1}}\right)+\bar{y}^{2} \operatorname{vâr}\left(\hat{\alpha_{2}}\right)+2 x \operatorname{cov} \hat{\operatorname{var}}\left(\hat{\alpha_{0}}, \hat{\alpha_{1}}\right) \\
+2 \bar{y} \operatorname{covar}\left(\hat{\alpha_{0}}, \hat{\alpha_{2}}\right)+2 x \bar{y} \operatorname{covar}\left(\hat{\alpha_{1}}, \hat{\alpha_{2}}\right), y \text { fixed at } \bar{y}, \\
\operatorname{varr}\left(\hat{\alpha_{0}}\right)+\hat{\sigma}_{a 0}^{2}+\bar{x}^{2} \operatorname{vâr}\left(\hat{\alpha_{1}}\right)+y^{2} \operatorname{vâr}\left(\hat{\alpha_{2}}\right)+2 \bar{x} \operatorname{cov} \operatorname{var}\left(\hat{\alpha_{0}}, \hat{\alpha_{1}}\right) \\
+2 y \operatorname{cov} \operatorname{ar}\left(\hat{\alpha_{0}}, \hat{\alpha_{2}}\right)+2 \bar{x} y \operatorname{covar}\left(\hat{\alpha_{1}}, \hat{\alpha_{2}}\right), x \text { fixed at } \bar{x} .
\end{array}\right. \\
=\left\{\begin{array}{l}
0.0300-0.0109 x+0.0068 x^{2} y \text { fixed at } \bar{y}, \\
0.0288-0.0054 y+0.0027 y^{2} x \text { fixed at } \bar{x} .
\end{array}\right. \\
\Rightarrow\left\{\begin{array}{c}
\text { s.e. }\left(\hat{\alpha_{0}}+\hat{a_{0 i}}+\hat{\alpha_{1}} x+\hat{\alpha_{2}} \bar{y}\right)=\sqrt{0.0300-0.0109 x+0.0068 x^{2}} \\
\text { s.e. }\left(\hat{\alpha_{0}}+\hat{a_{0 i}}+\hat{\alpha_{1}} \bar{x}+\hat{\alpha_{2}} y\right)=\sqrt{0.0288-0.0054 y+0.0027 y^{2}}
\end{array}\right.
\end{gathered}
$$

305 To calculate the standard errors and confidence intervals when one of the variables $x$ or $y$ was fixed, the mean of the fixed variable, $\bar{x}$ or $\bar{y}$, was substituted into the equations. 
At constant stocking 2 the two one-sided $95 \%$ confidence interval for the estimate of the exponent was calculated as

$$
\begin{aligned}
& \hat{\alpha_{0}}+\hat{a_{0 i}}+\hat{\alpha_{1}} x+\hat{\alpha_{2}} \bar{y} \pm t_{0.95,392} \times \text { s.e. }\left(\hat{\alpha_{0}}+\hat{a_{0 i}}+\hat{\alpha_{1}} x+\hat{\alpha_{2}} \bar{y}\right) \\
& =0.661+0.485 x+0.226 \bar{y} \pm 1.649 \times \sqrt{0.0300-0.0109 x+0.0068 x^{2}} \\
& =\left\{\begin{array}{l}
(0.567,1.129) \text { for the minimum age in decades, } 0.1, \\
(1.112,1.651) \text { for the maximum age in decades, } 1.2 .
\end{array}\right.
\end{aligned}
$$

At constant age 1 the two one-sided $95 \%$ confidence interval for the estimate of the exponent was calculated as

$$
\begin{aligned}
& \hat{\alpha_{0}}+\hat{a_{0 i}}+\hat{\alpha_{1}} \bar{x}+\hat{\alpha_{2}} y \pm t_{0.95,392} \times \text { s.e. }\left(\hat{\alpha_{0}}+\hat{a_{0 i}}+\hat{\alpha_{1}} \bar{x}+\hat{\alpha_{2}} y\right) \\
& =0.661+0.485 \bar{x}+0.226 y \pm 1.649 \times \sqrt{0.0288-0.0054 y+0.0027 y^{2}} \\
& =\left\{\begin{array}{l}
(0.692,1.235) \text { for the minimum } \mathrm{m}^{2} / \text { tree }, 0.375, \\
(0.839,1.371) \text { for the maximum } \mathrm{m}^{2} / \text { tree }, 1 .
\end{array}\right.
\end{aligned}
$$

\section{References}

Stage, A. R. (1963). A mathematical approach to polymorphic site index curves for

Grand fir. Forest Science, 9(2):167-180. 


\section{University Library}

\section{- M I N E R VA}

\section{A gateway to Melbourne's research publications}

Minerva Access is the Institutional Repository of The University of Melbourne

\section{Author/s:}

Capes, H;Maillardet, RJ;Baker, TG;Weston, CJ;McGuire, D;Dumbrell, IC;Robinson, AP

Title:

The Allometric Quarter-Power Scaling Model and Its Applicability to Grand Fir and Eucalyptus Trees

\section{Date:}

2017-12-01

\section{Citation:}

Capes, H., Maillardet, R. J., Baker, T. G., Weston, C. J., McGuire, D., Dumbrell, I. C. \& Robinson, A. P. (2017). The Allometric Quarter-Power Scaling Model and Its Applicability to Grand Fir and Eucalyptus Trees. JOURNAL OF AGRICULTURAL BIOLOGICAL AND ENVIRONMENTAL STATISTICS, 22 (4), pp.562-584. https://doi.org/10.1007/ s13253-017-0292-7.

Persistent Link:

http://hdl.handle.net/11343/282655 